\title{
Machine Learning Aided Static Malware Analysis: A Survey and Tutorial
}

\author{
Andrii Shalaginov, Sergii Banin, Ali Dehghantanha, Katrin Franke
}

\begin{abstract}
Malware analysis and detection techniques have been evolving during the last decade as a reflection to development of different malware techniques to evade network-based and host-based security protections. The fast growth in variety and number of malware species made it very difficult for forensics investigators to provide an on time response. Therefore, Machine Learning (ML) aided malware analysis became a necessity to automate different aspects of static and dynamic malware investigation. We believe that machine learning aided static analysis can be used as a methodological approach in technical Cyber Threats Intelligence (CTI) rather than resource-consuming dynamic malware analysis that has been thoroughly studied before. In this paper, we address this research gap by conducting an in-depth survey of different machine learning methods for classification of static characteristics of 32-bit malicious Portable Executable (PE32) Windows files and develop taxonomy for better understanding of these techniques. Afterwards, we offer a tutorial on how different machine learning techniques can be utilized in extraction and analysis of a variety of static characteristic of PE binaries and evaluate accuracy and practical generalization of these techniques. Finally, the results of experimental
\end{abstract}

Andrii Shalaginov

Norwegian Information Security Laboratory, Center for Cyber- and Information Security, Norwegian University of Science and Technology, Gjvik, Norway, e-mail: andrii . shalag inove ntnu.no

Sergii Banin

Norwegian Information Security Laboratory, Center for Cyber- and Information Security, Norwegian University of Science and Technology, Gjvik, Norway,e-mail: sergi i.baninentnu . no

Ali Dehghantanha

School of Computing, Science and Engineering, University of Salford, UK e-mail: a. dehghantanhaesalford.ac.uk

Katrin Franke

Norwegian Information Security Laboratory, Center for Cyber- and Information Security, Norwegian University of Science and Technology, Gjvik, Norway, e-mail: katrin. franke@ntnu. no 
study of all the method using common data was given to demonstrate the accuracy and complexity. This paper may serve as a stepping stone for future researchers in cross-disciplinary field of machine learning aided malware forensics.

\section{Introduction}

Stealing users' personal and private information has been always among top interests of malicious programs [8]. Platforms which are widely used by normal users have always been best targets for malware developers [9].

Attackers have leveraged malware to target personal computers [22], mobile devices [61], cloud storage systems [13], Supervisory Control and Data Acquisition Systems (SCADA) [12], Internet of Things (IoT) network [81] and even big data platforms [67].

Forensics examiners and incident handlers on the other side have developed different techniques for detection of compromised systems, removal of detected malicious programs [23, 14], network traffic [63], and even log analysis [69]. Different models have been suggested for detection, correlation and analyses of cyber threats [20] (on a range of mobile devices [43] and mobile applications [45], cloud applications [11], cloud infrastructure [46] and Internet of Things networks [47]). Windows users are still comprising majority of Internet users hence, it is not surprising to see Windows as the most adopted PC Operating System (OS) on top of the list of malware targeted platforms [73]. In response, lots of efforts have been made to secure Windows platform such as educating users [54, 25], embedding an antivirus software [40], deploying anti-malware and anti-exploitation tools [53, 52], and limiting users applications privilege [41].

In spite of all security enhancements, many malware are still successfully compromising Windows machines [36, 73] and malware is still ranked as an important threat to Windows platforms [33]. As result, many security professionals are still required to spend a lot of time on analyzing different malware species [10]. This is a logical step since malware analysis plays a crucial role in Cyber Threats Intelligence (CTI). There has been proposed a portal to facilitate CTI and malware analysis through interactive collaboration and information fusion [56].

There are two major approaches for malware analysis namely static (code) and dynamic (behavioral) malware analysis [16, 8]. In dynamic malware analysis, samples are executed and their run time behavior such as transmitted network traffic, the length of execution, changes that are made in the file system, etc. are used to understand the malware behavior and create indications of compromise for malware detection [16]. However, dynamic analysis techniques can be easily evaded by malware that are aware of execution conditions and computing environment [34]. Dynamic malware analysis techniques can only provide a snapshot view of the malware behavior and hence very limited in analysis of Polymorph or Metamorph species [44]. Moreover, dynamic malware analysis techniques are quite resource hungry which limits their enterprise deployment [37].

In static malware analysis, the analyst is reversing the malware code to achieve a deeper understanding of the malware possible activities. [28]. Static analysis relies 
on extraction of a variety of characteristics from the binary file such that function calls, header sections, etc. [83]. Such characteristics may reveal indicators of malicious activity that are going to be used in CTI [57]. However, static analysis is quite a slow process and requires a lot of human interpretation and hence [8].

Static analysis of PE32 is a many-sided challenge that was studied by different authors. Static malware analysis also was used before for discovering interconnections in malware species for improved Cyber Threat Intellifence [42, 66]. As 32-bit malware are still capable of infecting 64-bit platforms and considering there are still many 32-bit Windows OS it is not surprising that still majority of Windows malware are 32-bit Portable Executable files [8]. To authors knowledge there has not been a comparative study of ML-based static malware using a single dataset which produces comparable results. We believe that utilization of ML-aided automated analysis can speed up intelligent malware analysis process and reduce human interaction required for binaries processing. Therefore, there is a need for thorough review of the relevant scientific contributions and offer a taxonomy for automated static malware analysis.

The remainder of this paper is organized as follows. We first offer a comprehensive review of existing literature in machine learning aided static malware analysis. We believe this survey paves the way for further research in application of machine learning in static malware analysis and calls for further development in this field. Then, taxonomy of feature construction methods for variety of static characteristics and corresponding ML classification methods is offered. Afterwards, we offer a tutorial that applies variety of set of machine learning techniques and compares their performance. The tutorial findings provide a clear picture of pros and cons of MLaided static malware analysis techniques. To equally compare all the methods we used one benign and two malware datasets to evaluate all of the studied methods. This important part complements the paper due to the fact that most of the surveyed works used own collections, sometimes not available for public access or not published at all. Therefore, experimental study showed performance comparison and other practical aspects of ML-aided malware analysis. Section 4 gives an insight into a practical routine that we used to establish our experimental setup. Analysis of results and findings are given in the Section 5. Finally, the paper is concluded and several future works are suggested in the Section 6.

\section{An overview of Machine Learning-aided static malware detection}

This section provides an analysis of detectable static properties of 32 bit PE malware followed by detailed description of different machine learning techniques to develop a taxonomy of machine learning techniques for static malware analysis. 


\subsection{Static characteristics of PE files}

PE file format was introduced in Windows 3.1 as PE32 and further developed as PE32+ format for 64 bit Windows Operating Systems. PE files contain a Common Object File Format (COFF) header, standard COFF fields such as header, section table, data directories and Import Address Table (IAT). Beside the PE header fields a number of other static features can be extracted from a binary executable such as strings, entropy and size of various sections.

To be able to apply Machine Learning PE32 files static characteristics should be converted into a machine-understandable features. There exist different types of features depending on the nature of their values such that numerical that describes a quantitative measure (can be integer, real or binary value) or nominal that describes finite set of categories or labels. An example of the numerical feature is CPU (in \%) or RAM (in Megabytes) usage, while nominal can be a file type (like *.dll or *.exe) or Application Program Interface (API) function call (like write() or read()).

1. n-grams of byte sequences is a well-known method of feature construction utilizing sequences of bytes from binary files to create features. Many tools have been developed for this purpose such as hexdump [39] created 4-grams from byte sequences of PE32 files. The features are collected by sliding window of $n$ bytes. This resulted in 200 millions of features using 10-grams for about two thousands files in overall. Moreover, feature selection (FS) was applied to select 500 most valuable features based on Information Gain metric. Achieved accuracy on malware detection was up to $97 \%$ using such features. Another work on byte n-grams [51] described usage of 100-500 selected n-grams yet on a set of 250 malicious and 250 benign samples. Similar approach [31] was used with $10, \ldots, 10,000$ best $n$-grams for $n=1, \ldots, 10$. Additionally, ML methods such that Naive Bayes, C4.5, k-NN and others were investigated to evaluate their applicability and accuracy. Finally, a range of 1-8 n-grams [27] can result in 500 best selected n-grams that are used later to train AdaBoost and Random Forests in addition to previously mentioned works.

2. Opcode sequences or operation codes are set of consecutive low level machine abstractions used to perform various CPU operations. As it was shown [62] such features can be used to train Machine Learning methods for successful classification of the malware samples. However, there should be a balance between the size of the feature set and the length of n-gram opcode sequence. $\mathrm{N}$-grams with the size of 4 and 5 result in highest classification accuracy as unknown malware samples could be unveiled on a collection of 17,000 malware and 1,000 benign files with a classification accuracy up to $94 \%$ [58]. Bragen [6] explored reliability of malware analysis using sequences of opcodes based on the 992 PE-files malware and benign samples. During the experiments, about 50 millions of opcodes were extracted. 1-gram- and 2-gram-based features showed good computational results and accuracy. Wang et al. [79] presented how the 2-tuple opcode sequences can be used in combination with density clustering to detect malicious or benign files. 
3. API calls are the function calls used by a program to execute specific functionality. We have to distinguish between System API calls that are available through standard system DLLs and User API calls provided by user installed software. These are designed to perform a pre-defined task during invocation. Suspicious API calls, anti-VM and anti-debugger hooks and calls can be extracted by PE analysers such as PEframe [4]. [83] studied 23 malware samples and found that some of the API calls are present only in malwares rather than benign software. Function calls may compose in graphs to represent PE32 header features as nodes, edges and subgraphs [84]. This work shows that ML methods achieve accuracy of $96 \%$ on 24 features extracted after analysis of 1,037 malware and 2,072 benign executables. Further, in [71] 20,682 API calls were extracted using PE parser for 1,593 malicious and benign samples. Such large number of extracted features can help to create linearly separable model that is crucial for many ML methods as Support Vector Machines (SVM) or single-layer Neural Networks. Another work by [55] described how API sequences can be analysed in analogy with byte $\mathrm{n}$-grams and opcode $\mathrm{n}$-grams to extract corresponding features to classify malware and benign files. Also in this work, an array of API calls from IAT (PE32 header filed) was processed by Fisher score to select relevant features after analysis of more than $34 \mathrm{k}$ samples.

4. PE header represents a collection of meta data related to a Portable Executable file. Basic features that can be extracted from PE32 header are Size of Header, Size of Uninitialized Data, Size of Stack Reserve, which may indicate if a binary file is malicious or benign [15]. The work [76] utilized Decision Trees to analyse PE header structural information for describing malicious and benign files. [77] used 125 raw header characteristics, 31 section characteristics, 29 section characteristics to detect unknown malware in a semi-supervised approach. Another work [80] used a dataset containing 7,863 malware samples from Vx Heaven web site in addition to 1,908 benign files to develop a SVM based malware detection model with accuracy of 98\%. [38] used F-score as a performance metric to analyse PE32 header features of 164,802 malicious and benign samples. Also [29] presented research of two novel methods related to PE32 string-based classier that do not require additional extraction of structural or meta-data information from the binary files. Moreover, [84] described application of 24 features along with API calls for classification of malware and benign samples from VxHeaven and Windows XP SP3 respectively. Further, ensemble of features was explored [59], where authors used in total 209 features including structural and raw data from PE32 file header. Further, Le-Khac et al. [35] focused on Control Flow Change over first 256 addresses to construct n-gram features.

In addition to study of specific features used for malware detection, we analyzed articles devoted to application of ML for static malware analysis published between 2000 and 2016, which covers the timeline of Windows NT family that are still in use as depicted in the Figure 1. We can see that the number of papers that are relevant to our study is growing from 2009 and later, which can be justified on the basis of increase in the number of Windows users (potential targets) and corresponding malware families. 


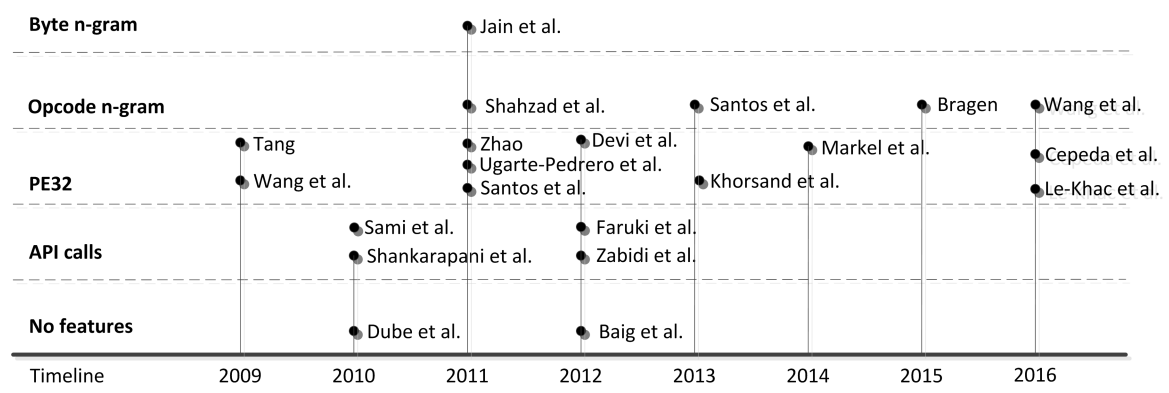

Fig. 1: Timeline of works since 2009 that involved static analysis of Portable Executable files using method characteristics using also ML method for binary malware classification

Challenges. Despite the fact that some of the feature construction techniques reflected promising precision of $90+\%$ in differentiation between malicious and benign executables, there are still no best static characteristic that guarantee $100 \%$ accuracy of malware detection. This can be explained by the fact that malware are using obfuscation and encryption techniques to subvert detection mechanisms. In addition, more accurate approaches such as bytes N-GRAMS are quite resource intensive and hardly practical in the real world.

\subsection{Machine Learning methods used for static-based malware detection}

\subsubsection{Statistical methods}

Exploring large amounts of binary files consists of statistical features may be simplified using so called frequencies or likelihood of features values. These methods are made to provide prediction about the binary executable class based on statistics of different static characteristics (either automatically or manually collected) which are applicable to malware analysis too as describe by Shabtai et al. [60]. To process such data, extract new or make predictions the following set of statistical methods can be used:

Naive Bayes is a simple probabilistic classifier, which is based on Bayesian theorem with naive assumptions about independence in correlation of different features. The Bayes Rule can be explained as a following conditional independences of features values with respect to a class:

$$
P\left(C_{k} \mid V\right)=P\left(C_{k}\right) \frac{P\left(V \mid C_{k}\right)}{P(V)}
$$


where $P\left(C_{k}\right)$ is a prior probability of class $C_{k}, k=1, \ldots, m_{0}$ which is calculated from collected statistics according to description of variables provided by Kononenko et al. [32]. This method is considered to tackle just binary classification problem (benign against malicious) since it was originally designed as multinomial classifier. $V=\left\langle v_{1}, \ldots, v_{a}\right\rangle$ is the vector of attributes values that belongs to a sample. In case of Naive Bayes input values should be symbolical, for example strings, opcodes, instruction n-grams etc. $P(V)$ is the prior probability of a sample described with vector $V$. Having training data set and given vector $V$ we count how many samples contain equal values of attributes (e.g. based on the number of sections or given opcode sequence). It is important to mention that $V$ have not to be of length of full attribute vector and can contain only one attribute value. $P\left(V \mid C_{k}\right)$ is the conditional probability of a sample described with $V$ given the class $C_{k}$. And $P\left(C_{k} \mid V\right)$ conditional probability of class $C_{k}$ with $V$. Based on simple probability theory we can describe conditional independence of attribute values $v_{i}$ given the class $C_{k}$ :

$$
P\left(V \mid C_{k}\right)=P\left(v_{1} \wedge \ldots \wedge v_{a} \mid C_{k}\right)=\prod_{i=1}^{a} P\left(v_{i} \mid C_{k}\right)
$$

Dropping the mathematical operations we get final version of Equation 1:

$$
P\left(C_{k} \mid V\right)=P\left(C_{k}\right) \prod_{i=1}^{a} \frac{P\left(C_{k} \mid v_{i}\right)}{P\left(C_{k}\right)}
$$

So, the task of this machine learning algorithm is to calculate conditional and unconditional probabilities as described in the Equation 3 using a training dataset. To be more specific, the Algorithm 1 pseudo code shows the calculation of the conditional probability. 


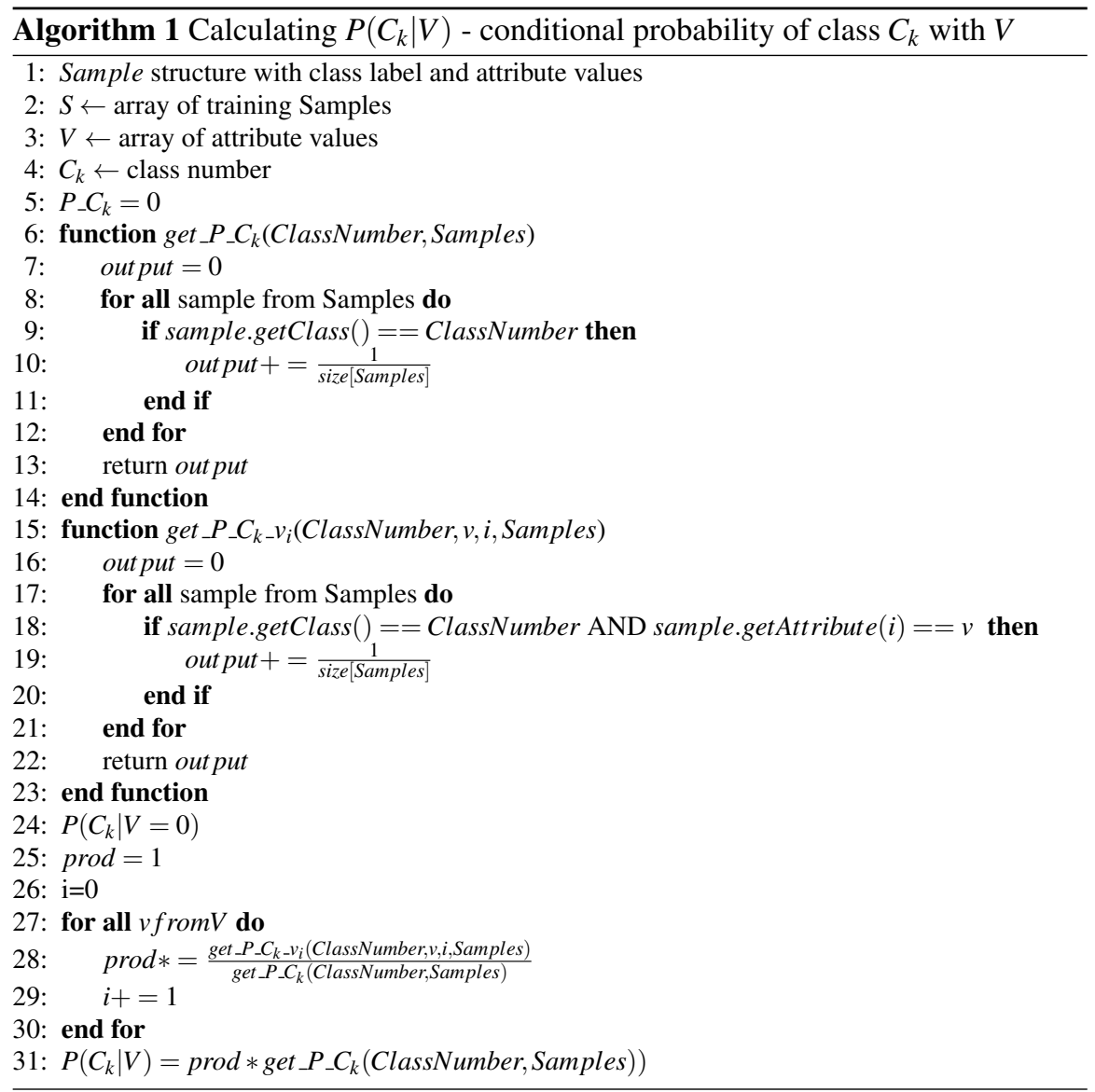

So, we can see from the Equation 1 that given output is as a probability that a questioned software sample belongs to one or another class. Therefore, the classification decision will be made by finding a maximal value from set of corresponding class likelihoods. Equation 4 provides formula that assigns class label to the output:

$$
\hat{y}=\underset{k \in\{1, \ldots, K\}}{\operatorname{argmax}} P\left(C_{k}\right) P\left(V \mid C_{k}\right) .
$$

Bayesian Networks is a probabilistic directed acyclic graphical model (sometimes also named as Bayesian Belief Networks), which shows conditional dependencies using directed acyclic graph. Network can be used to detect "update knowledge of the state of a subset of variables when other variables (the evidence variables) are observed" [32]. Bayesian Networks are used in many cases of classification and information retrieval (such as semantic search). The method's routine can be described as following. If edge goes from vertex $A$ to vertex $B$, then $\mathrm{A}$ is a parent of $\mathrm{B}$, and $\mathrm{B}$ is an ancestor of $\mathrm{A}$. If from $A$ there is oriented path to another 
vertex $B$ exists then $B$ is ancestor of $A$, and $A$ is a predecessor of $B$. Lets designate set of parent vertexes of vertex $V_{i}$ as parents $\left(V_{i}\right)=P A_{i}$. Direct acyclic graph is called Bayesian Network for probability distribution $P(v)$ given for set of random variables $V$ if each vertex of graph has matched with random variable from $V$. And edge of a graph fits next condition: every variable $v_{i}$ from $V$ must be conditionally independent from all vertexes that are not its ancestors if all its direct parents $P A_{i}$ are initialized in graph $G$ :

$$
\forall V_{i} \in V \Rightarrow P\left(v_{i} \mid p a_{i}, s\right)=P\left(v_{i} \mid p a_{i}\right)
$$

where $v_{i}$ is a value of $V_{i}, S$ - set of all vertexes that are not ancestors of $V_{i}, s$ configuration of $S, p a_{i}$ - configuration of $P A_{i}$. Then full general distribution of the values in vertexes could be written as product of local distributions, similarly to Naive Bayes rules:

$$
\mathrm{P}\left(V_{1}, \ldots, V_{n}\right)=\prod_{i=1}^{n} \mathrm{P}\left(V_{i} \mid \operatorname{parents}\left(V_{i}\right)\right)
$$

Bayesian Belief Networks can be used for classification [32], thus can be applied for malware detection and classification as well [58]. To make Bayesian Network capable of classification it should contain classes as parent nodes which don't have parents themselves. Figure 2 shows an example of such Bayesian network.

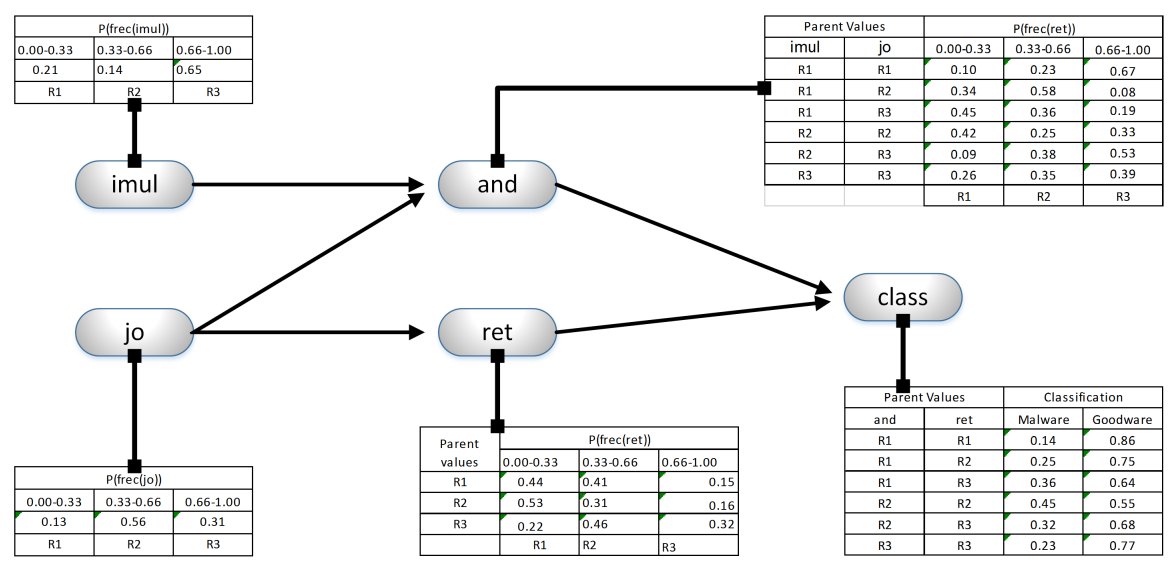

Fig. 2: Bayesian network suitable for malware classification [58]

\subsubsection{Rule based}

Rule based algorithms are used for generating crisp or inexact rules in different Machine Learning approaches [32]. The main advantage of having logic rules involved 
in malware classification is that logical rules that operate with statements like equal, grater then, less or equal to can be executed on the hardware level which significantly increases the speed of decision making.

C4.5 is specially proposed by Quinlan [50] to construct decision trees. These trees can be used for classification and especially for malware detection [75]. The process of trees training includes processing of previously classified dataset and on each step looks for an attribute that divides set of samples into subsets with the best information gain. $\mathrm{C} 4.5$ has several benefits in compare with other decision tree building algorithms:

- Works not only with discrete but with continuous attributes as well. For continuous attributes it creates threshold tp compares values against [49].

- Take into account missing attributes values.

- Works with attributes with different costs.

- Perform automate tree pruning by going backward through the tree and removing useless branches with leaf nodes.

Algorithm 2 shows a simplified version of decision tree building algorithm.

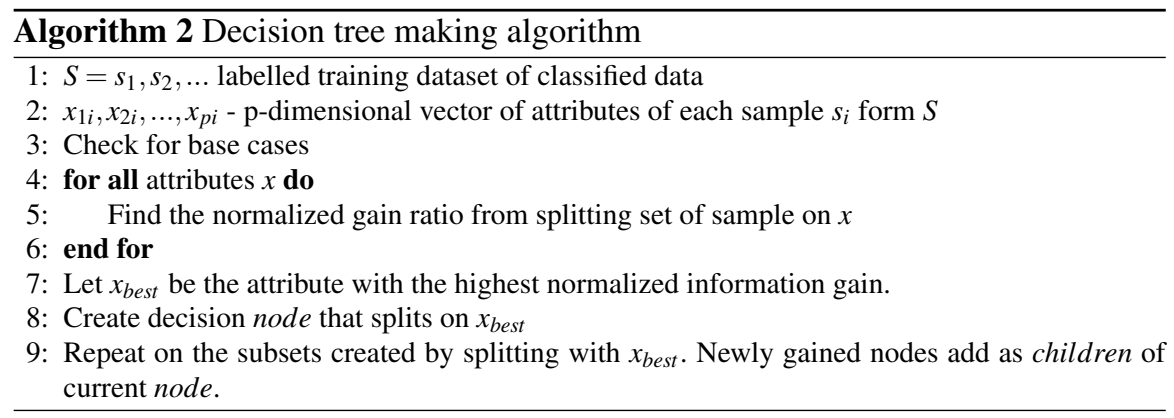

Neuro-Fuzzy is a hybrid models that ensembles neural networks and fuzzy logic to create human-like linguistic rules using the power of neural networks. Neural network also known as artificial neural network is a network of simple elements which are based on the model of perceptron [65]. Perceptron implements previously chosen activation functions which take input signals and their weights and produces an output, usually in the range of $[0,1]$ or $[-1,1]$. The network can be trained to perform classification of complex and high-dimensional data. Neural Networks are widely used for classification and pattern recognition tasks, thus for malware analysis [72]. The problem is that solutions gained by Neural Networks are usually impossible to interpret because of complexity of internal structure and increased weights on the edges. This stimulates usage of Fuzzy Logic techniques, where generated rules are made in human-like easy-interpretable format: IF $X>3$ AND $X<5$ THEN $Y=7$.

Basic idea of Neuro-Fuzzy (NF) model is a fuzzy system that is trained with a learning algorithm similar to one from neural networks theory. NF system can be 
represented as a neural network which takes input variables and produces output variables while connection weights are represented as encoded fuzzy sets. Thus at any stage (like prior to, in process of and after training) NF can be represented as a set of fuzzy rules. Self-Organising (Kohonen) maps [30] is the most common techniques of combining Neuro and Fuzzy approaches. Shalaginov et al. [64] showed the possibility of malware detection using specially-tuned Neuro-Fuzzy technique on a small dataset. Further, NF showed good performance on large-scale binary problem of network traffic analysis [63]. This method has also proven its efficiency on a set of multinomial classification problems. In particular, it is useful when we are talking about distinguishing not only "malware" or "goodware" but also detecting specific type of "malware" [68]. Therefore, it has been improved for the multinational classification of malware types and families by Shalaginov et al. [70].

\subsubsection{Distance based}

This set of methods is used for classification based on predefined distance measure. Data for distance-based methods should be carefully prepared, because computational complexity grows significantly with space dimensionality (number of features) and number of training samples. Thus there is a need for proper feature selection as well as sometimes for data normalization.

k-Nearest Neighbours or k-NN is classification and regression method. k-NN does not need special preparation of the dataset or actual "training" as the algorithm is ready for classification right after labelling the dataset. The algorithm takes a sample that is need to be classified and calculates distances to samples from training dataset, then it selects $k$ nearest neighbours (with shortest distances) and makes decision based on class of this $k$ nearest neighbours. Sometimes it makes decision just on the majority of classes in this $k$ neighbours selection, while in other cases there is weights involved in process of making decision. When k-NN is used for malware classification and detection there is a need for careful feature selection as well as a methodology for dealing with outliers and highly mixed data, when training samples cannot create distinguishable clusters [58].

Support Vector Machine or SVM is a supervised learning method. It constructs one or several hyperplanes to divide dataset for classification. Hyperplane is constructed to maximize distance from it to the nearest data points. Sometimes kernel transformation is used to simplify hyperplanes. Building a hyperplane is usually turned into two-class problem (one vs one, one vs many) and involves quadratic programming. Lets have linearly separable data (as shown in Figure 3 ) which can be represented as $\mathscr{D}=\left\{\left(\mathbf{x}_{i}, y_{i}\right) \mid \mathbf{x}_{i} \in \mathbb{R}^{p}, y_{i} \in\{-1,1\}\right\}_{i=1}^{n}$. Where $y_{i}$ is 1 or -1 depending on class of point $x_{i}$. Each $x_{i}$ is p-dimensional vector (not always normalised). The task is to find hyperplane with maximum margin that divides dataset on points with $y_{i}=1$ and $y_{i}=-1: w \cdot x-b=0$. Where $w$ is a normal vector to a hyperplane [21]. If dataset is linearly separable we can build two hyperplanes $w \cdot x-b=1$ and $w \cdot x-b=-1$ between which there will be no (or in case of soft margin maximal allowed number) points. Distance between them (margin) is $\frac{2}{\|w\|}$, so to maximize 
margin we need to minimize $\|w\|$ and to find parameters of hyperplane we need to introduce Lagrangian multipliers $\alpha$ and solve Equation 7 with quadratic programming techniques.

$$
\arg \min _{\mathbf{w}, b} \max _{\alpha \geq 0}\left\{\frac{1}{2}\|\mathbf{w}\|^{2}-\sum_{i=1}^{n} \alpha_{i}\left[y_{i}\left(\mathbf{w} \cdot \mathbf{x}_{\mathbf{i}}-b\right)-1\right]\right\}
$$

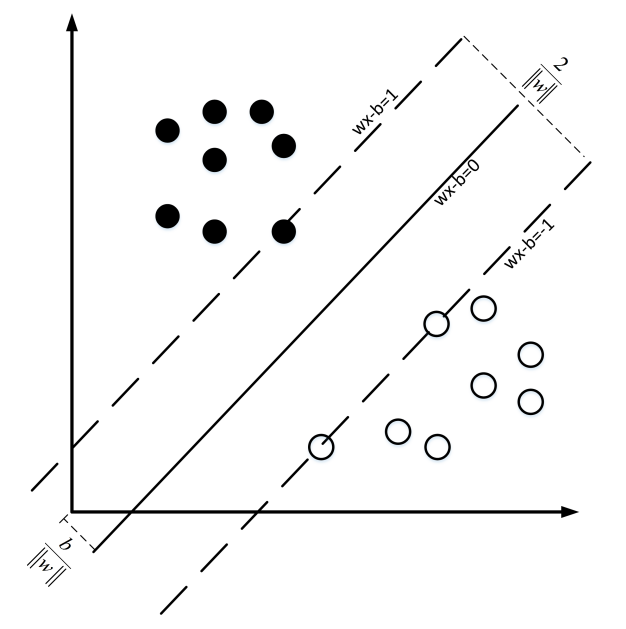

Fig. 3: Maximum margin hyperplane for two class problem [32]

Sometimes there is a need to allow an algorithm to work with misclassified data hence leaving some points inside the margin based on the degree of misclassification $\xi$. So the Equation 3 turns into Equation 8.

$$
\begin{array}{r}
\arg \min _{\mathbf{w}, \xi, b} \max _{\alpha, \beta}\left\{\frac{1}{2}\|\mathbf{w}\|^{2}+C \sum_{i=1}^{n} \xi_{i}-\sum_{i=1}^{n} \alpha_{i}\left[y_{i}\left(\mathbf{w} \cdot \mathbf{x}_{\mathbf{i}}-b\right)-1+\xi_{i}\right]-\sum_{i=1}^{n} \beta_{i} \xi_{i}\right\} \\
\text { with } \alpha_{i}, \beta_{i} \geq 0
\end{array}
$$

Also the data might be linearly separated, so there is a need for kernel trick. The basic idea is to substitute every dot product with non-linear kernel function. Kernel function can be chosen depending on situation and can be polynomial, Gaussian, hyperbolic etc. SVM is a very powerful technique which can give good accuracy if properly used, so it often used in malware detection studies as shown by Ye et al. [82]. 


\subsubsection{Neural networks}

Neural Network is based on the model of perceptron which has predefined activation function. In the process of training weights of the links between neurons are trained to fit train data set with minimum error with use of back propagation. Artificial Neural network (ANN) consists of input layer, hidden layer (layers) and output layer as it is shown on Figure 4.

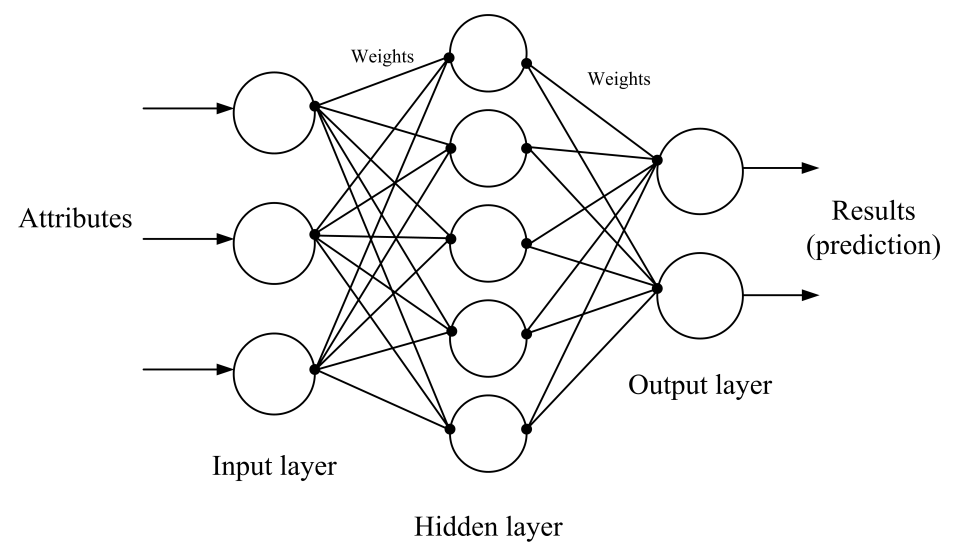

Fig. 4: Artificial neural network [32]

The input layer takes normalized data, while hidden output layer produces activation output using neuron's weighted input and activation function. Activation function is a basic property of neuron that takes input values given on the input edges, multiply them by weights of these edges and produces output usually in a range of $[0,1]$ or $[-1,1]$. Output layer is needed to present results and then interpret them. Training of ANN starts with random initialization of weights for all edges. Then feature vector of each sample is used as an input. Afterwards, result gained on the output layer is compared to the real answers. Any errors are calculated and using back-propagation all weights are tuned. Training can continue until reaching desired number of training cycles or accuracy. Learning process of ANN can be presented as shown in the Algorithm 3. Artificial Neural networks can be applied for complex models in high-dimensional spaces. This is why it often used for malware research [72]. 


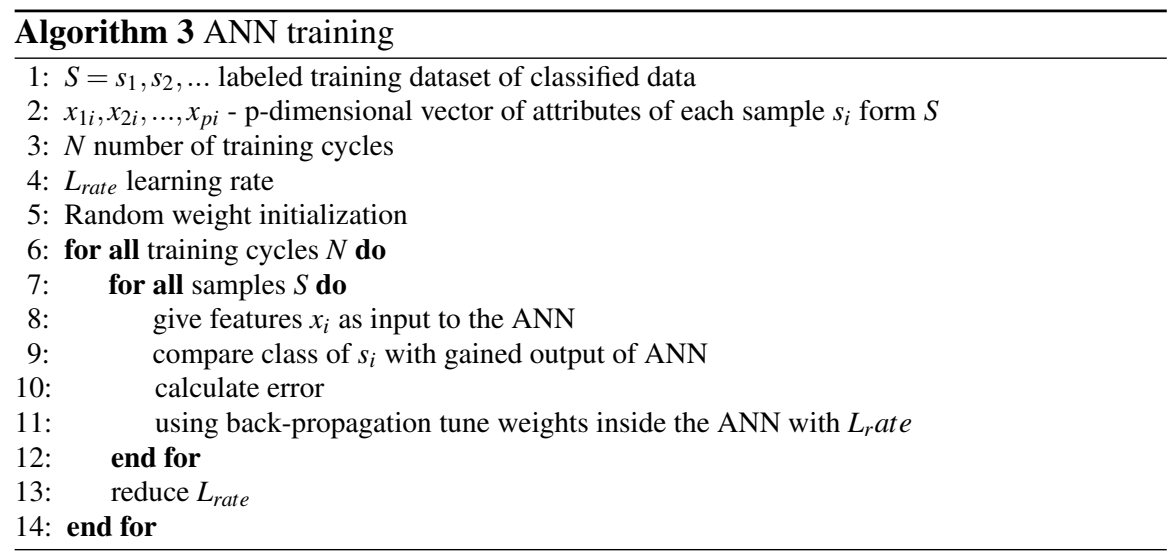

\subsubsection{Open Source and Freely available ML Tools}

Today machine learning is widely used in many areas of research with many publicly available tools (Software products, libraries etc.).

Weka or Waikato Environment for Knowledge Analysis is a popular, free, cross platform and open source tool for machine learning. It supports many of popular ML methods with possibility of fine tuning of the parameters and final results analysis. It provides many features such as splitting dataset and graphical representation of the results. Weka results are saved in .arff file which is specially prepared CSV file with header. It suffers from couple of issues including no support for multi-thread computations and poor memory utilization especially with big datasets.

Python weka wrapper is the package which allows using power of Weka through Python programs. It uses javabridge to link Java-based Weka libraries to python. It provides the same functionality as Weka, but provides more automation capacities.

LIBSVM and LIBLINEAR are open source ML libraries written in C++ supporting kernelized SVMs for linear, classification and regression analysis. Bindings for Java, Mathlab and R are also present. It uses space-separated files as input, where zero values need not to be mentioned.

RapidMiner is machine learning and data mining tool with a user friendly GUI and support for a lot of ML and data mining algorithms.

Dlib is a free and cross-platform C++ toolkit which supports different -machine learning algorithms and allows multi-threading and utilization of Python APIs.

\subsubsection{Feature Selection \& Construction process}

Next important step after the characteristics extraction is so-called Feature Selection process [32]. Feature Selection is a set of methods that focus on elimination of irrelevant or redundant features that are not influential for malware classification. 
This is important since the number of characteristics can be extremely large, while only a few can actually be used to differentiate malware and benign applications with a high degree of confidence. The most common feature selection methods are Information Gain, and Correlation-based Feature Subset Selection (CFS) [24]. The final goal of Feature Selection is to simplify the process of knowledge transfer from data to a reusable classification model.

\subsection{Taxonomy of malware static analysis using Machine Learning}

Our extensive literature study as reflected in Table 1 resulted to proposing a taxonomy for malware static analysis using machine learning as shown in Figure 5. Our taxonomy depicts the most common methods for analysis of static characteristics, extracting and selecting features and utilizing machine learning classification techniques. Statistical Pattern Recognition process [26] was used as the basis for our taxonomy modelling.

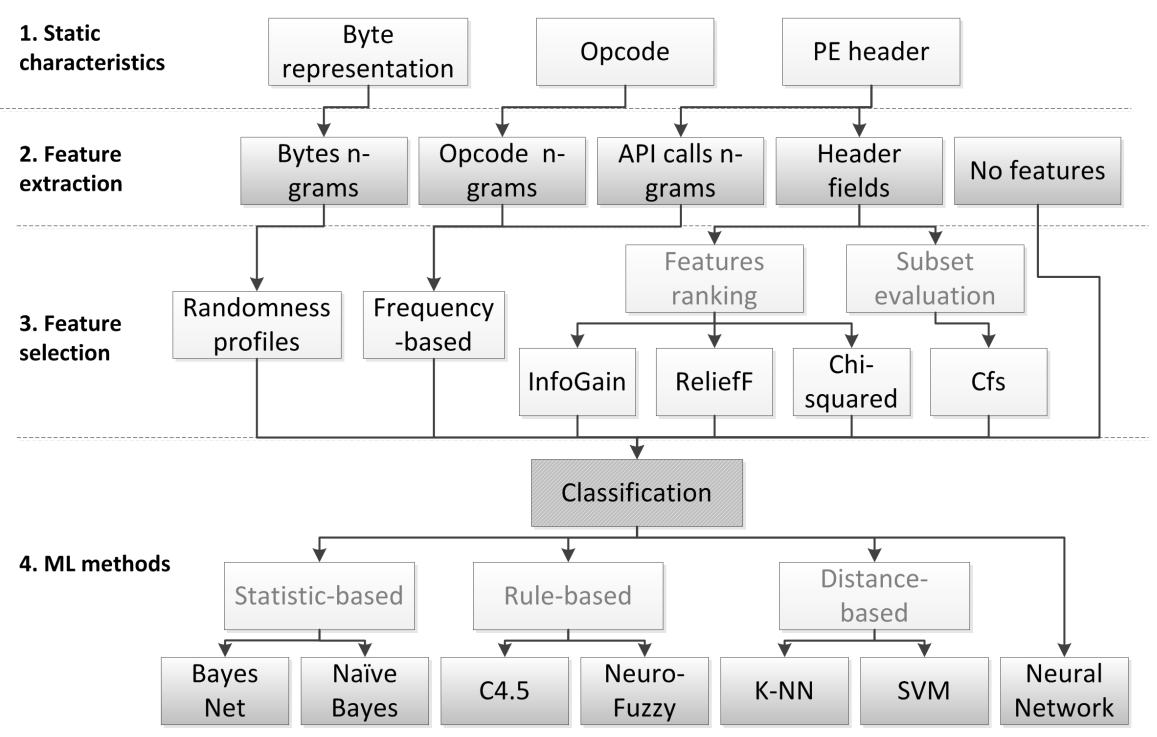

Fig. 5: Taxonomy of common malware detection process based on static characteristics and Machine Learning

\begin{tabular}{|c|c|c|c|c|}
\hline Year Authors & Dataset & Features & FS & ML \\
\hline & & PE32 header & & \\
\hline
\end{tabular}




\begin{tabular}{|c|c|c|c|c|c|}
\hline \multirow{3}{*}{2016} & \multirow{3}{*}{$\begin{array}{l}\text { Cepeda } \\
\text { al. [7] }\end{array}$} & \multirow{3}{*}{$\begin{array}{l}\text { et 7,630 malware and } 1.818 \\
\text { goodware }\end{array}$} & \multirow{3}{*}{$\begin{array}{l}57 \text { features from } \\
\text { VirisTotal }\end{array}$} & \\
\hline & & & & & \\
\hline & & & & \multicolumn{2}{|c|}{$\begin{array}{l}\text { with } 9 \\
\text { features } \\
\text { finally }\end{array}$} \\
\hline 2016 & $\begin{array}{l}\text { Le-Khac } \\
\text { al. [35] }\end{array}$ & $\begin{array}{l}\text { et Malicious: } 94 \text {;Benign: } \\
620\end{array}$ & $\begin{array}{l}\text { Control } \begin{array}{r}\text { Flow } \\
\text { Change and } \\
\text { n-grams }\end{array} \text { 2-6 } \\
\end{array}$ & - & Naive Bayes \\
\hline 2014 & $\begin{array}{l}\text { Markel et al. } \\
{[38]}\end{array}$ & $\begin{array}{l}\text { 1. Malicious: } 122,799, \mathrm{Be}- \\
\text { nign: } 42,003\end{array}$ & $\begin{array}{l}46 \text { features use } \\
\text { python 'pefile' }\end{array}$ & - & $\begin{array}{l}\text { Naive Bayes, Logistic } \\
\text { Regression, Classifica- } \\
\text { tion and Regression Tree } \\
\text { (CART) }\end{array}$ \\
\hline 2013 & $\begin{array}{l}\text { Khorsand et al. } \\
\text { [29] }\end{array}$ & $\begin{array}{l}\text { 1. Benign: } 850 \text { EXE and } \\
750 \text { DLL; Malware: } 1600 \\
\text { from VX heavens }\end{array}$ & eliminated & - & $\begin{array}{l}\text { Prediction by partial } \\
\text { matching }\end{array}$ \\
\hline 2012 & Devi et al. [15] & $\begin{array}{l}4,075 \text { PE files: } 2954 \text { ma- } \\
\text { licious and } 1121 \text { Win- } \\
\text { dows XP SP2 benign }\end{array}$ & $2+5$ features & - & $\begin{array}{l}\text { BayesNet, k-NN, SVM, } \\
\text { AdaBoostM1, Decision } \\
\text { table, C4.5, Random } \\
\text { Forest, Random Tree }\end{array}$ \\
\hline 2011 & Zhao [84] & $\begin{array}{l}3109 \text { PE: } 1037 \text { viruses } \\
\text { from Vx Heavens and } \\
2072 \text { benign executable } \\
\text { on Win XP Sp3 }\end{array}$ & $\begin{array}{l}24 \text { features from PE } \\
\text { files using Control } \\
\text { Flow Graph-based } \\
\text { on nodes }\end{array}$ & & $\begin{array}{l}\text { Random Forest, Decision } \\
\text { Tree, Bagging, C4.5 }\end{array}$ \\
\hline 2011 & $\begin{array}{l}\text { Ugarte- } \\
\text { Pedrero } \\
\text { al. [77] }\end{array}$ & $\begin{array}{l}500 \text { benign from WinXP } \\
\text { et and } 500 \text { non-packed from } \\
\text { Vxheaven; } 500 \text { packed }+ \\
500 \text { Zeus }\end{array}$ & $\begin{array}{l}166 \text { structure fea- } \\
\text { tures of PE file }\end{array}$ & InfoGain & $\begin{array}{l}\text { Learning with Local and } \\
\text { Global Consistency, Ran- } \\
\text { dom Forest }\end{array}$ \\
\hline 2011 & $\begin{array}{l}\text { Santos } \\
\text { al. [59] }\end{array}$ & $\begin{array}{l}500 \text { benign and } 500 \\
\text { malicious from VxHeav- } \\
\text { ens, also packed and not } \\
\text { packed }\end{array}$ & $\begin{array}{l}209 \text { structural fea- } \\
\text { tures }\end{array}$ & InfoGain & Collective Forest \\
\hline 2009 & Tang [76] & $\begin{array}{l}361 \text { executables and } 449 \\
\text { normal trojan files }\end{array}$ & $\begin{array}{l}\text { PE header structural } \\
\text { features }\end{array}$ & & Decision Tree \\
\hline 2009 & $\begin{array}{l}\text { Wang } \\
\text { al. [80] }\end{array}$ & $\begin{array}{l}\text { et Benign: 1,908, Mali- } \\
\text { cious: } 7,863\end{array}$ & $\begin{array}{l}143 \text { PE header en- } \\
\text { tries }\end{array}$ & $\begin{array}{l}\text { InfoGain, } \\
\text { Gain raio }\end{array}$ & SVM \\
\hline \multicolumn{6}{|c|}{ bytes $n$-gram sequences } \\
\hline 2011 & Jain et al. [27] & $\begin{array}{l}1,018 \text { malware and } 1,120 \\
\text { benign samples }\end{array}$ & $\begin{array}{l}1-8 \text { byte, n-gram, } \\
\text { best n-gram by } \\
\text { documentwise } \\
\text { frequency }\end{array}$ & & $\begin{array}{l}\text { NB, iBK, J48, Ad- } \\
\text { aBoost1, RandomForest }\end{array}$ \\
\hline 2007 & $\begin{array}{l}\text { Masud } \\
\text { al. [39] }\end{array}$ & $\begin{array}{l}\text { 1st set }-1,435 \text { executa- } \\
\text { bles: } 597 \text { of which are be- } \\
\text { nign and } 838 \text { are mali- } \\
\text { cious. } 2 \text { nd set }-2,452 \text { ex- } \\
\text { ecutables: } 1,370 \text { benign } \\
\text { and } 1,082 \text { malicious }\end{array}$ & 500 best n-grams & InfoGain & SVM \\
\hline 2006 & $\begin{array}{l}\text { Reddy } \\
\text { al. [51] }\end{array}$ & $\begin{array}{l}250 \text { malware vs } 250 \text { be- } \\
\text { nign }\end{array}$ & $\begin{array}{l}100-500 \\
\text { n-gram }\end{array}$ & $\begin{array}{l}\text { Document } \\
\text { Fre- } \\
\text { quency, } \\
\text { InfoGain }\end{array}$ & \\
\hline 2004 & $\begin{array}{l}\text { Kolter } \\
\text { al. [31] }\end{array}$ & $\begin{array}{l}\text { et } 1971 \text { benign, } 1651 \text { mali- } \\
\text { cious from Vx Heaven }\end{array}$ & 500 best n-grams & InfoGain & Naive Bayes, SVM, C4.5 \\
\hline \multicolumn{6}{|c|}{ opcode $n$-gram sequences } \\
\hline 2016 & $\begin{array}{l}\text { Wang } \\
\text { al. [79] }\end{array}$ & $\begin{array}{l}11,665 \text { malware and } \\
1,000 \text { benign samples }\end{array}$ & $\begin{array}{l}\text { 2-tuple opcode se- } \\
\text { quences }\end{array}$ & $\begin{array}{l}\text { informatior } \\
\text { entropy }\end{array}$ & density clustering \\
\hline 2015 & Bragen [6] & $\begin{array}{l}992 \text { malwares, } 771 \text { be- } \\
\text { nign from Windows Vista }\end{array}$ & $\begin{array}{l}\text { 1-4 n-gram opcode } \\
\text { with vocabulary } \\
530-714,390\end{array}$ & $\begin{array}{l}\text { Cfs, Chi- } \\
\text { sqaured, } \\
\text { InfoGain, } \\
\text { ReliefF, } \\
\text { SymUncert }\end{array}$ & $\begin{array}{l}\text { Random Forest, C4.5, } \\
\text { Naive Bayes, bayes Net, } \\
\text { Baggin, ANN, SOM, } \\
\text { k-nn } \\
\text { t. }\end{array}$ \\
\hline 2013 & $\begin{array}{l}\text { Santos } \\
\text { al. [58] }\end{array}$ & $\begin{array}{l}\text { et } 13,189 \text { malware } \\
13,000 \text { benign }\end{array}$ & top 1,000 features & InfoGain & $\begin{array}{l}\text { Random Forest, J48, } \\
\text { k-Nearest Neighbours, } \\
\text { Bayesian networks, SVM }\end{array}$ \\
\hline 2011 & $\begin{array}{l}\text { Shahzad } \\
\text { al. [62] }\end{array}$ & $\begin{array}{l}\text { Benign: } 300 \text {, Malicious: } \\
300 \text { on Windows XP }\end{array}$ & $\begin{array}{l}\text { coabulary of } 1,413 \\
\text { with } n \text {-gram }=4\end{array}$ & tf-idf & $\begin{array}{l}\text { ZeroR, Ripper, C4.5, } \\
\text { SVM, Naive Bayes, k-nn }\end{array}$ \\
\hline
\end{tabular}




\begin{tabular}{|c|c|c|c|c|c|}
\hline \multicolumn{6}{|c|}{ API calls } \\
\hline 2012 & $\begin{array}{l}\text { Zabidi } \\
\text { al. [83] }\end{array}$ & 23 malware and 1 benign & $\begin{array}{l}\text { API calls, debugger } \\
\text { features, VM fea- } \\
\text { tures }\end{array}$ & & - \\
\hline 2012 & $\begin{array}{l}\text { Faruki } \\
\text { al. [19] }\end{array}$ & $\begin{array}{l}3234 \text { benign, } 3256 \text { mal- } \\
\text { ware }\end{array}$ & 1-4 API call-gram & - & $\begin{array}{l}\text { Random Forest, SVM, } \\
\text { ANN, C4.5, Naive bayes }\end{array}$ \\
\hline & $\begin{array}{l}\text { Shankarapani } \\
\text { et al. [71] }\end{array}$ & $\begin{array}{l}1593 \text { PE files: } 875 \text { benign } \\
\text { and } 715 \text { malicious }\end{array}$ & API calls sequence & - & SVM \\
\hline 2010 & Sami et al. [55] & $\begin{array}{l}34,820 \text { PE: } 31,869 \text { ma- } \\
\text { licious and } 2951 \text { benign } \\
\text { from Windows }\end{array}$ & API calls & $\begin{array}{l}\text { Fisher } \\
\text { Score }\end{array}$ & $\begin{array}{l}\text { Random Forest, C4.5, } \\
\text { Naive Bayes }\end{array}$ \\
\hline \multicolumn{6}{|c|}{ no features / not described } \\
\hline 2012 & Baig et al. [5] & $\begin{array}{l}200 \text { packed PE and } 200 \\
\text { unpacked from Windows } \\
7 \text {, Windows } 2003 \text { Server }\end{array}$ & file entropy & - & - \\
\hline 2010 & Dube et al. [17] & $\begin{array}{l}40,498 \text { samples: } 25,974 \\
\text { malware, } 14,524 \text { benign }\end{array}$ & from 32 bit files & - & Decision Tree \\
\hline
\end{tabular}

Table 1: Analysis of ML methods applicability for different types of static characteristics

To get a clear picture on application domain of each machine learning and feature selection method we analysed reported performance as shown in Figure 6.Majority of researchers were using byte n-gram, opcode n-gram and PE32 header fields for static analysis while C4.5, SVM or k-NN methods were mainly used for malware detection. Information Gain is the prevalent method to define malware attributes. Also we can see that n-gram-based method tend to use corresponding set of feature selection like tf-idf and Symmetric Uncertainty that are more relevant for large number of similar sequences. On the other hand, PE32 header-based features tend to provide higher entropies for classification and therefore Control-Flow graph-based and Gain Ratio are more suitable for this task.

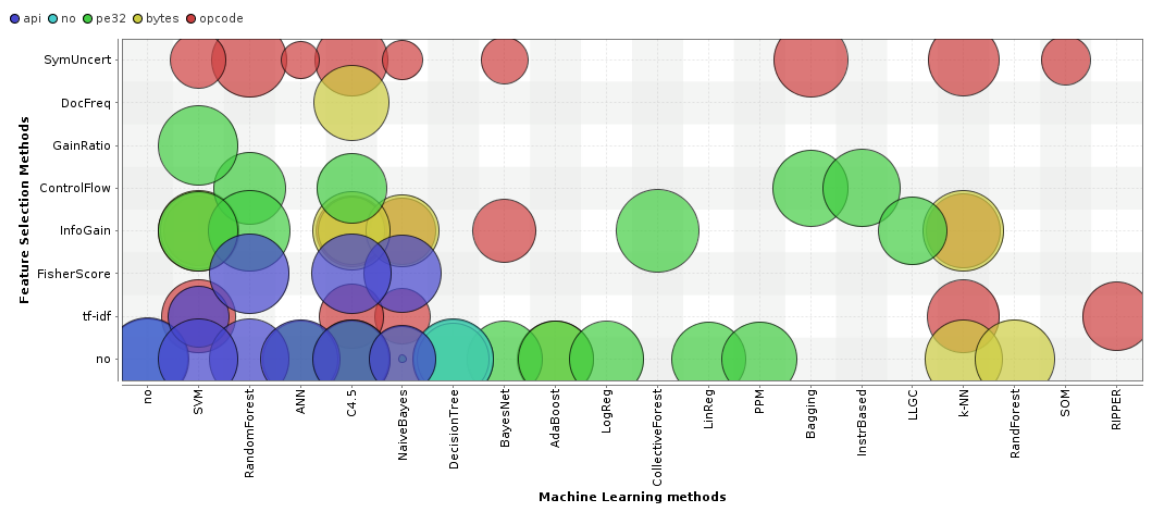

Fig. 6: Comparison of accuracy of various static characteristics with respect to feature selection and machine learning methods. Colour of the bubbles shows used characteristics for detection, while size of the bubble denotes achieved accuracy 
To conclude, one can say that majority of authors either extract features that offers good classification accuracy, or use conventional methods like Information Gain. However, n-gram based characteristics need other FS approaches to eliminate irrelevant features. Rule-based ML is the most commonly used classification method along with SVM. Forest-based method tends to be more applicable for PE32 header-based features. Also ANN is not commonly-used technique. While most of the works achieved accuracies of $80-100 \%$, some Bayes-based methods offered much lower accuracy even down to $50 \%$ only.

\section{Approaches for Malware Feature Construction}

Similar the previous works, following four sets of static properties are suggested for feature classification in this paper:

PE32 header features characterize the PE32 header information using the $P E$ frame tools [77]. Following numerical features will be used in our experiments:

- ShortInfo_Directories describes 16 possible data directories available in PE file. The most commonly used are "Import", "Export", "Resource", "Debug", "Relocation".

- ShortInfo_Xor indicates detected XOR obfuscation.

- ShortInfo_DLL is a binary flag of whether a file is executable or dynamicallylinked library.

- ShortInfo_FileSize measures size of a binary file in bytes.

- ShortInfo_Detected shows present techniques used to evade the detection by antiviruses like hooks to disable execution in virtualized environment or suspicious API calls.

- ShortInfo_Sections is a number of subsections available in the header.

- DigitalSignature contains information about the digital signatture that can be present in a file

- Packer describes used packer detected by PEframe

- AntiDebug gives insight into the techniques used to prevent debugging process.

- AntiVM is included to prevent the execution in a virtualize environment.

- SuspiciousAPI indicates functions calls that are labelled by PEframe as suspicious.

- SuspiciousSections contains information about suspicious sections like ".rsrc \u0000 \u0000 \u0000"

- $U r l$ is a number of different url addresses found in the binary file.

Byte n-gram. N-gram is a sequence of some items (with minimum length of 1) that are predefined as minimal parts of the object expressed in Bytes. By having the file represented as a sequence of bytes we can construct 1-gram, 2-gram, 3-gram etc. $\mathrm{N}$-grams of bytes, or byte n-grams are widely used as features for machine learning and static malware analysis [27, 51]. 
Reddy et al. [51] used n-grams of size 2,3 and 4 with combination of SVM, Instance-based learner and Decision Tree algorithms to distinguish between malicious and benign executables. After extracting n-grams they used class-wise document frequency as a feature selection measure and showed that class-wise document frequency is performing better than Information Gain as a feature selection measure. Jain et al. [27] used n-grams in range of 1 to 8 as features and Naive Bayes, Instance Based Learner and AdaBoost1 [3] as machine learning algorithms for malware classification and reported byte 3 -grams as the best technique.

Opcode n-gram represent a set of instructions that will be performed on the CPU when binary is executed. These instructions are called operational codes or opcodes. To extract opcodes from executable we need to perform disassembly procedure. After this opcodes will be represented as short instructions names such as POP, PUSH, $M O V, A D D, S U B$ etc. Santos et al. [58] described a method to distinguish between malicious and benign executables or detecting different malware families using opcode sequences of length 1 to 4 using Random Forest, J48, k-Nearest Neighbours, Bayesian Networks and Support Vector Machine algorithms [3].

API calls is a set of tools and routines that help to develop a program using existent functionality of an operating system. Since most of the malware samples are platform dependent it is very much likely that their developers have use APIs as well. Therefore, analysing API calls usage among benign and malicious software can help to find malware-specific API calls and therefore are suitable to be used as a feature for machine learning algorithms. For example, [71] successfully used Support Vector Machines with frequency of API calls for malware classification. [78] provided a methodology for classification of malicious and benign executables using API calls and n-grams with n from 1 to 4 and achieved accuracy of 97.23\% for 1-gram features. [19] used so-called API call-gram model with sequence length ranging from 1-4 and reached accuracy of $97.7 \%$ was achieved by training with 3grams. In our experiments we are going to use 1 and $2 \mathrm{n}$-grams as features generated from API calls.

\section{Experimental Design}

All experiments were conducted on a dedicated Virtual machine (VM) on Ubuntu 14.04 server running on Xen 4.4. The server had an Intel(R) Core(TM) i7-3820 CPU @ $3.60 \mathrm{GHz}$ with 4 cores ( 8 threads), out of which 2 cores (4 threads) were provided to the VM. Disk space is allocated on the SSD RAID storage based on Samsung 845DC. Installed server memory was Kingston PC-1600 RAM, out of which 8GB was available for the VM. Operating system was an Ubuntu 14.0464 bit running on ae dedicated VM together with all default tools and utilities available in the OS's repository. Files pre-processing were performed using bash scripts due to native support in Linux OS. To store extracted features we utilised MySQL 5.5 database engine together with Python v 2.7.6 and PHP v 5.5.9 connectors. 
For the experiments we used a set of benign and malicious samples. To authors knowledge there have not been published any large BENIGN SOFTWARE REFERENCE DATASETS, so we have to create our own set of benign files. Since the focus of the paper is mainly on PE32 Windows executables, we decided to extract corresponding known-to-be-good files from different versions of MS Windows, including different software and multimedia programs installations that are available. The OSes that we processed were 32 bit versions of Windows XP, Windows 7, Windows 8.1 and Windows 10. Following two Windows malware datasets were used in our research:

1. VX HEAVEN [2] dedicated to distribute information about the computer viruses and contains 271,092 sorted samples dating back from 1999.

2. VIRUS SHARE [1] represent sharing resource that offers $29,119,178$ malware samples and accessible through VirusShare tracker as of 12th of July, 2017. We utilized following two archives: VirusShare_00000.zip created on 2012-06-15 00:39:38 with a size of 13.56 GB and VirusShare_00207.zip created on 201512-16 22:56:17 with a size of $13.91 \mathrm{~GB}$, all together contained 131,072 unique, uncategorised and unsorted malware samples. They will be referred further as malware_000 and malware_207.

To be able to perform experiments on the dataset, we have to filter out irrelevant samples (not specific PE32 and not executables), which are out of scope in this paper. However, processing of more than 100k samples put limitations and require non-trivial approaches to handle such amount of files. We discovered that common ways of working with files in directory such that simple $l s$ and $m v$ in bash take unreasonable amount of time to execute. Also there is no way to distinguish files by extension like *.dll or *.exe since the names are just $m d 5$ sums. So, following filtering steps were performed:

1. Heap of unfiltered malware and benign files were placed into two directories "malware/" and "benign/".

2. To eliminate duplicates, we renamed all the files to their MD5 sums.

3. PE32 files were detected in each folder using file Linux command:

$\backslash \$$ file 000000 b4dccfbaa5bd981af2c1bbf59a

000000b4dccfbaa5bd981af2c1bbf59a: PE32 executable (

DLL) (GUI) Intel 80386, for MS Windows

4. All PE32 files from current directory that meet our requirement were scrapped and move to a dedicated one:

\#! / bin/sh

cd .. / windows 1 ;

counter $=0$;

for i in $*$; do

counter $=\$(($ counter +1$))$;

echo "\$counter";

VAR=" fi l e $\left.\lrcorner \$ i_{\lrcorner} \mid\right\lrcorner$grep $\left.\lrcorner P E 32\right\lrcorner "$; 


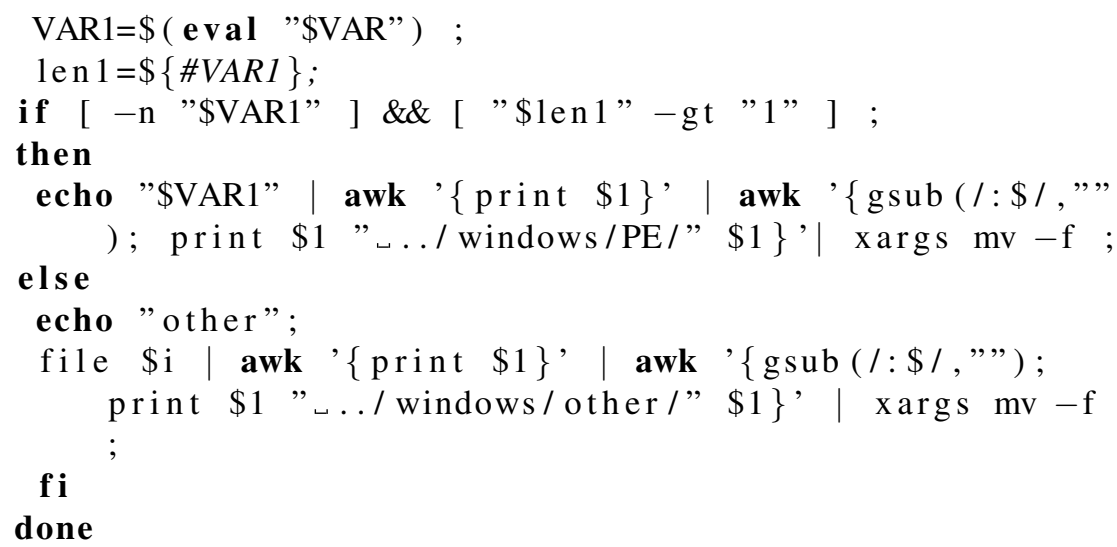

5. We further can see a variety of PE32 modifications for 32bit architecture:

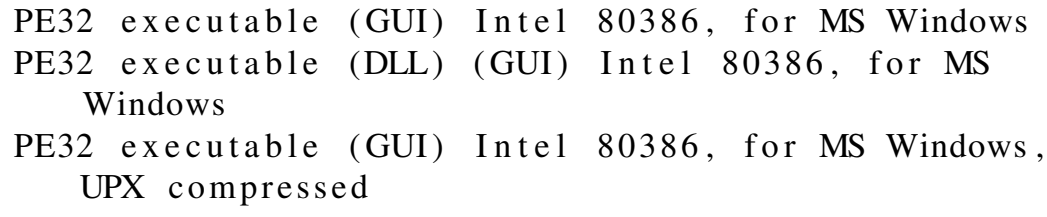

Following our purpose to concentrate on 32bit architecture, only PE32 are filtered out from all possible variants of PE32 files shown about.

6. After extracting a target group of benign and malicious PE32 files, multiple rounds of feature extraction are performed according to methods used in the literature.

7. Finally, we insert extracted features into the corresponding MySQL database to ease the handling, feature selection and machine learning processes respectively.

After collecting all possible files and performing the pre-processing phase, we ended up with the sets represented in the Table 2.

Table 2: Characteristics of the dataset collected and used for our experiments after filtering PE files

\begin{tabular}{lrr}
\hline Dataset & Number of files & Size \\
\hline Benign & 16,632 & $7.4 \mathrm{~GB}$ \\
Malware_000 & 58,023 & $14.0 \mathrm{~GB}$ \\
Malware_207 & 41,899 & $16.0 \mathrm{~GB}$ \\
\hline
\end{tabular}

Further, feature construction and extraction routine from PE files was performed using several tools as follows:

1. PEFRAme [4] is an open source tool specifically designed for static analysis of PE malware. It extracts various information from PE header ranging from packers to anti debug and anti vm tricks. 
2. HexDump is a standard Linux command line tool which is used to display a file in specific format like ASCII or one-byte octal.

3. ОвJDUMP is a standard Linux command line too to detect applications instructions, consumed memory addresses, etc.

\section{Results \& Discussions}

Before testing different ML techniques for malware detection it is important to show that our datasets actually represent the real-world distribution of the malware and goodware. Comparison of "Compile Time" field of PE32 header can be utilized for this purpose. Figure 7 represents log-scale histogram of the compilation time for our benign dataset. Taking into consideration the Windows OS timeline we found a harmony between our benign dataset applications compile time and development of Microsoft Windows operating systems. To start with, Windows 3.1 was originally released on April 6, 1992 and our plot of benign applications indicates the biggest spike in early 1992. Later on in 1990th, Windows 95 was due on 24 August 1995, while next Windows 98 was announced on 25 June 1998. Further, 2000th marked release of Windows XP on October 25, 2001. Next phases on the plot correspond to the release of Windows Vista on 30th January 2007 and Windows 7 on 22nd October 2009. Next popular version (Windows 8) appeared on 26th October 2012 and the latest major spike in the end of 2014 corresponds to the release of Windows 10 on 29th July 2015.

Further, compilation time distribution for the first malware dataset malware_000 is given in the Figure 8. We can clearly see that release of newer Windows version is always followed by an increase of cumulative distribution of malware samples in following 6 to 12 month. It can be seen that the release of 32bit Windows 3.1 cause a spike in a number of malware. After this the number of malware compiled each year is constantly growing. Then, another increase can be observed in second half of the 2001 which corresponds to the release of the Windows XP and so on.

Considering the fact that MS DOS was released in 1981 it makes compilation times before this day look like fake or just obfuscated intentionally. On the other hand the dataset malware_000 cannot have dates later than June 2012. Therefore, malware with compilation time prior to 1981 or later than Jan 2012 are tampered

\subsection{Accuracy of ML-aided Malware Detection using Static Characteristics}

This part presents results of apply Naive Bayes, BayesNet, C4.5, k-NN, SVM, ANN and NF machine learning algorithms against static features of our dataset namely PE32 header, Bytes n-gram, Opcode n-gram, and API calls n-gram. 


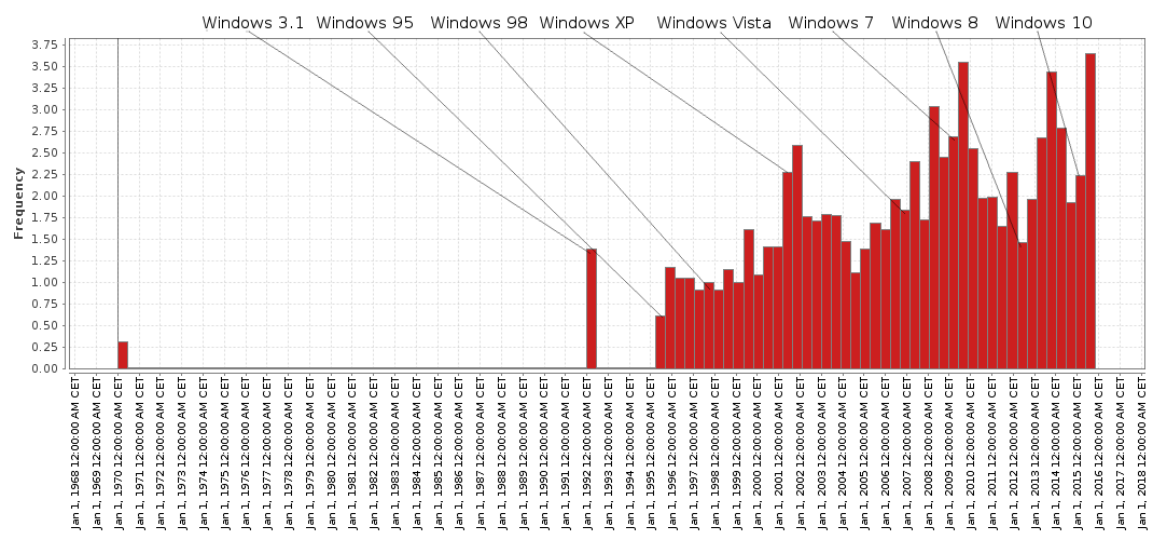

Fig. 7: Log-scale histogram of compilation times for benign dataset

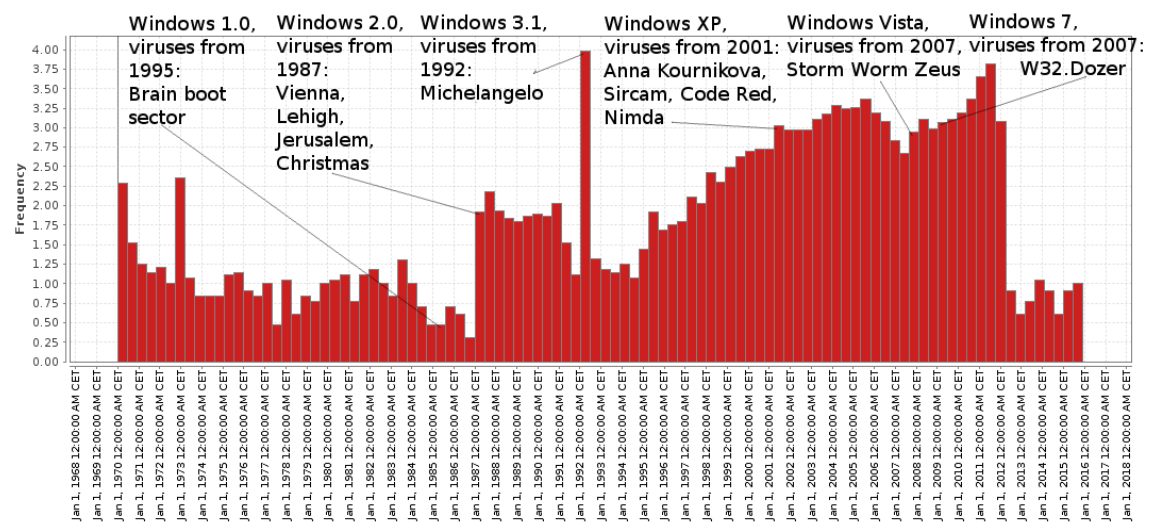

Fig. 8: Log-scale histogram of compilation times for malware_000 dataset

\subsubsection{PE32 header}

PE32 header is one of the most important features relevant to threat intelligence of PE32 applications. We performed feature selection using $C f s$ and InfoGain methods with 5-fold cross-validation as presented in the Table 3.

We can clearly see that the features from the Short Info section in PE32 headers can be used as a stand-alone malware indicators, including different epochs. Number of directories in this section as well as file size and flag of EXE or DLL have bigger merits in comparison to other features. To contrary, Anti Debug and Suspicious API sections from PEframe cannot classify a binary file. Finally, we can say that digital signature and Anti VM files in PE32 headers are almost irrelevant in malware detection. Further, we performed exploration of selected ML methods that 


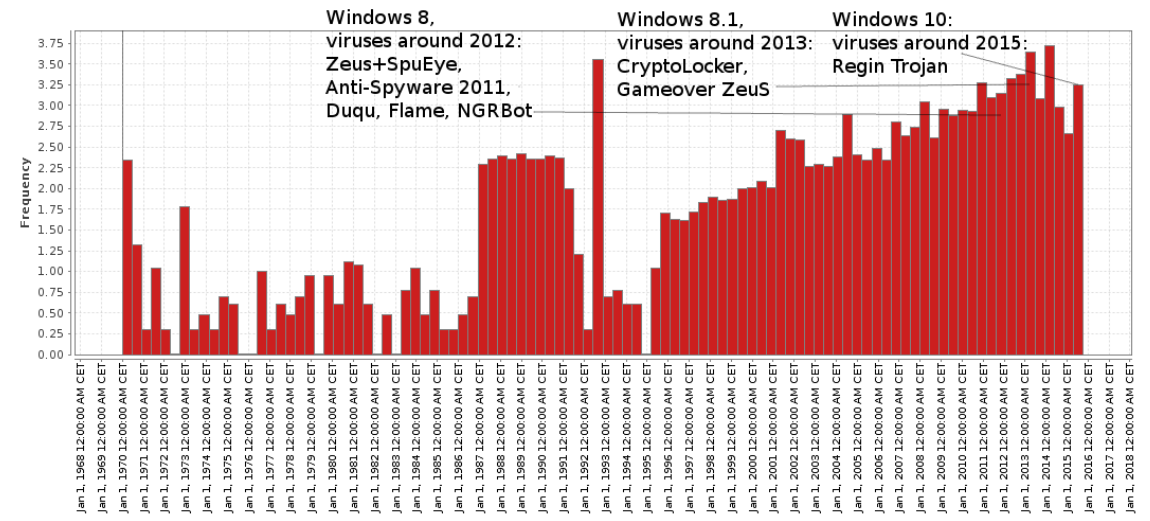

Fig. 9: Log-scale histogram of compilation times for malware_207 dataset

Table 3: Feature selection on PE32 features. Bold font denotes selected features according to InfoGain method

\begin{tabular}{|c|c|c|}
\hline Benign vs Malware_000 & Benign vs Malware_207 & Malware_000 vs Malware $\_207$ \\
\hline \multicolumn{3}{|c|}{ Information Gain } \\
\hline merit attribute & merit attribute & merit attribute \\
\hline $\begin{array}{l}\text { 0.377 } \text { ShortInfo_Directories } \\
0.278 \text { ShortInfo_DLL } \\
\text { 0.118 } \text { AntiDebug } \\
0.099 \text { Packer } \\
\text { 0.088 } \text { SuspiciousSections } \\
\text { 0.082 } \text { ShortInfo_Xor } \\
\text { 0.076 SuspiciousAPI } \\
\text { 0.045 } \text { ShortInfo_FileSize } \\
\text { 0.034 ShortInfo_Detected } \\
\text { 0.022 } \text { Url } \\
\text { 0.004 } \text { AntiVM } \\
\text { 0 ShortInfo_Sections } \\
\text { 0 DigitalSignature }\end{array}$ & $\mid \begin{array}{ll}0.369 & \text { ShortInfo_DLL } \\
0.252 & \text { ShortInfo_Directories } \\
0.142 & \text { ShortInfo_FileSize } \\
0.105 & \text { SuspiciousSections } \\
\text { 0.101 } & \text { SuspiciousAPI } \\
\text { 0.089 } & \text { AntiDebug } \\
\text { 0.084 } & \text { ShortInfo_Detected } \\
\text { 0.054 } & \text { ShortInfo_Xor } \\
\text { 0.050 } & \text { Packer } \\
\text { 0.036 } & \text { Url } \\
\text { 0.002 } & \text { AntiVM } \\
\text { 0 } & \text { ShortInfo_Sections } \\
\text { 0 } & \text { DigitalSignature }\end{array}$ & $\mid \begin{array}{ll}0.131 & \text { ShortInfo_FileSize } \\
0.094 & \text { ShortInfo_Detected } \\
0.064 & \text { SuspiciousAPI } \\
0.044 & \text { ShortInfo_Directories } \\
0.036 & \text { Packer } \\
0.028 & \text { AntiDebug } \\
0.017 & \text { SuspiciousSections } \\
0.016 & \text { Url } \\
0.015 & \text { AntiVM } \\
0.012 & \text { ShortInfo_Xor } \\
0.002 & \text { ShortInfo_DLL } \\
\text { 0 } & \text { ShortInfo_Sections } \\
\text { 0 } & \text { DigitalSignature }\end{array}$ \\
\hline \multicolumn{3}{|c|}{ Cfs } \\
\hline attribute & attribute & attribute \\
\hline $\begin{array}{l}\text { ShortInfo_Directories } \\
\text { ShortInfo_Xor } \\
\text { ShortInfo_DLL } \\
\text { ShortInfo_Detected } \\
\text { Url }\end{array}$ & $\begin{array}{l}\text { ShortInfo_Directories } \\
\text { ShortInfo_Xor } \\
\text { ShortInfo_DLL }\end{array}$ & $\begin{array}{l}\text { ShortInfo_Directories } \\
\text { ShortInfo_FileSize } \\
\text { ShortInfo_Detected } \\
\text { Packer }\end{array}$ \\
\hline
\end{tabular}

can be used with selected features. By extracting corresponding numerical features mentioned earlier, we were able to achieve classification accuracy levels presented in Table 4. Table 3 presents also accuracy of ML method after performing feature selection. Here we used whole sub-sets defined by $C f s$ method and features with merit of $\geq 0.1$ detected by InfoGain. 
Table 4: Comparative classification accuracy based on features from PE32 header, in \%. Bn, Ml_000 and Ml_207 are benign and two malaware datasets respectively

\begin{tabular}{|c|c|c|c|c|}
\hline Dataset & Naive Bayes & BayesNet C4.5 & k-NN SVM ANN & NF \\
\hline \multicolumn{5}{|c|}{ All features } \\
\hline Bn vs Ml_000 & 90.29 & $91.42 \mathbf{9 7 . 6 3}$ & 97.3087 .7595 .08 & 92.46 \\
\hline Bn vs Ml_207 & 88.27 & $91.21 \mathbf{9 6 . 4 3}$ & $\begin{array}{ll}95.99 & 84.88 \quad 93.24\end{array}$ & 89.03 \\
\hline Ml_000 vs Ml_207 & 63.41 & $71.59 \mathbf{8 2 . 4 5}$ & 82.1173 .7769 .99 & 69.01 \\
\hline \multicolumn{5}{|c|}{ Information Gain } \\
\hline Bn vs Ml_000 & 88.32 & 89.1794 .09 & 94.0194 .0993 .51 & 87.53 \\
\hline Bn vs Ml_207 & 87.25 & 90.3995 .06 & $94.58 \quad 84.5592 .37$ & 87.88 \\
\hline Ml_000 vs Ml_207 & 58.26 & 67.0567 .77 & $\mathbf{7 0 . 7 0} 69.4663 .19$ & 51.31 \\
\hline \multicolumn{5}{|c|}{ Cfs } \\
\hline Bn vs Ml_000 & 89.35 & $90.89 \mathbf{9 5 . 3 9}$ & 95.3895 .1693 .69 & 85.85 \\
\hline Bn vs Ml_207 & 86.88 & 89.6791 .61 & 91.6891 .6891 .68 & 81.91 \\
\hline Ml_000 vs Ml_207 & 67.45 & 70.9576 .98 & $\begin{array}{lll}76.92 & 72.15 & 68.18\end{array}$ & 67.06 \\
\hline
\end{tabular}

Malware and goodware can be easily classified using full set as well as sub-set of features. One can notice that ANN and C4.5 performed much better than other methods. It can be also seen that the high quality of these features made them very appropriate to differentiate between the benign and malware_000 dataset. Further, we can see that the two datasets malware_000 and malware_207 are similar and extracted features do not provide a high classification accuracy. Neural Network was used with 3 hidden layers making it a non-linear model and experiments were performed using 5-fold cross-validation technique.

\subsubsection{Bytes n-gram}

Bytes n-gram is a very popular method for static analysis of binary executables. This method has one significant benefit: in order to perform analysis there is no need of previous knowledge about file type and internal structure since we use its raw (binary) form. For feature construction we used random profiles that were first presented by Ebringer et al. [18] called fixed sample count (see Figure 10), which generates fixed number of random profiles regardless of the file size and sliding window algorithm. In this method each file is represented in a hexadecimal format and frequencies of each byte are counted to build a Huffman tree for each file. Then using window of fixed size and moving it on fixed skip size the randomness profile of each window is calculated. A Randomness profile is sum of Huffman code length of each byte in a single window. The lower the randomness in a particular window the bigger will be the randomness of that profile.

We chose 32 bytes as the most promising sliding window size $[18,48,74]$ and due to big variety of file sizes in our dataset, we chose 30 best features (or pruning size in terminology from [18]) which are the areas of biggest randomness (the most unique parts) in their original order. This features was fed into different machine 


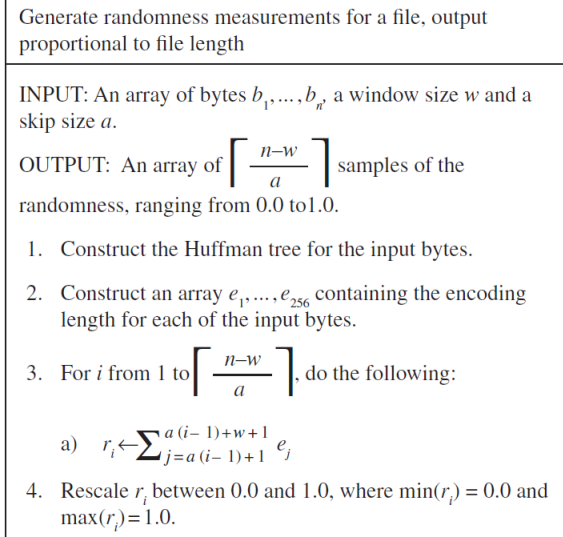

Fig. 10: Sliding window algorithm [18]

learning algorithms as shown in Table 5. Our results indicate that the accuracy of this technique is not that high as it was originally developed to preserve local details of a file ( [18])while the size of file affects localness a lot. In our case file sizes vary from around $0.5 \mathrm{~Kb}$ to $53.7 \mathrm{Mb}$ which adversely affect the results. Despite worse results it is still easier to distinguish between benign executables and malware than between malware from different time slices. Also we can see that ANN is better in Benign vs Malware_000 dataset, C4.5 in Benign vs Malware_207 and Malware_000 vs Malware_207 datasets.

Table 5: Classification accuracy based on features from bytes n-gram randomness profiles, in \%

\begin{tabular}{llllllll}
\hline Dataset & \multicolumn{7}{c}{ Naive Bayes BayesNet } \\
\hline \multicolumn{7}{c}{ All features } \\
\hline Bn vs Ml_000 & 69.9 & 60.4 & 76.9 & 75.6 & 78.3 & 78.3 & 74.8 \\
Bn vs Ml_207 & 70.3 & 68.2 & 75.8 & 75.6 & 72.1 & 71.6 & 68.2 \\
M1_000 vs Ml_207 & 50.1 & 64.0 & 68.1 & 64.7 & 58.1 & 60.1 & 58.2 \\
\hline
\end{tabular}

Also it should be noted that we did not use feature selection methods as in the case of PE32 header features. Both Information gain and Cfs are not efficient due to the similarity of features and equivalence in importance for classification process. For the first dataset the Information Gain was in the range of 0.0473-0.0672 while for the second dataset it was in the range of 0.0672-0.1304 and for the last it was 0.0499-0.0725. Moreover, Cfs produces best feature subset nearly equal to full set. Therefore, we decided to use all features as there is no subset that could possibly be better than original one. 


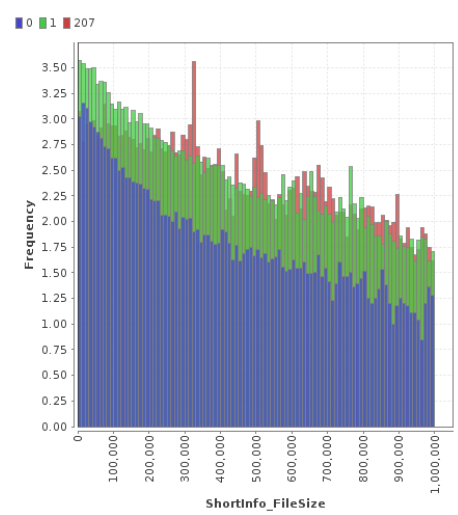

(a) Less than $1 \mathrm{M}$

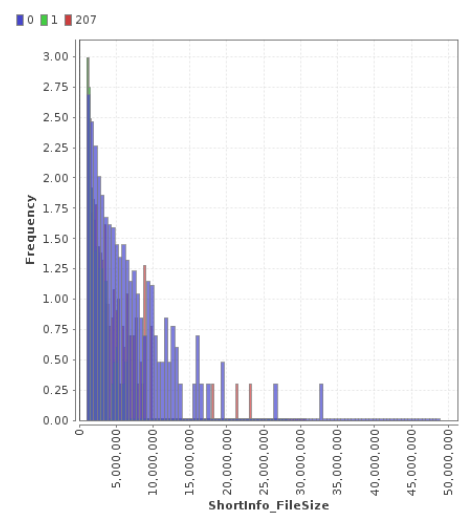

(b) Bigger than $1 \mathrm{M}$

Fig. 11: Distribution of file size values in Bytes for three classes

\subsubsection{Opcode n-gram}

Opcode n-gram consists of assembly instructions which construct the executable file. The main limitation of this method is that in order to gain opcodes we need disassemble an application which sometimes fails to give correct opcodes due to different anti-disassembly and packing techniques used in executables hence we filtered out this kind of files from our dataset. We extracted 100 most common 3- and 4-grams from each of three file sets in our dataset. Then we extracted a set of 200 most common n-grams - which are called feature n-grams - to build a presence vector where value 1 was assigned if a certain $n$-gram from feature $n$-grams is present in top 100 most used n-grams of the file. Table 6 represents results of feature selection performed on the dataset with 3-grams. As can be seen the first two pair of datasets have a lot of common n-grams, while selected n-grams for the third pair of dataset is totally different. For Information Gain the threshold of 0.1 was used for both benign and malware datasets, while for the last set we used InfoGain of 0.02 .

These data were passed to machine learning algorithms and results are shown in Tables 7 and 9. As can be seen C4.5 performed well and had the highest accuracy almost in all experiments. Also feature selection significantly reduced the number of n-grams from 200 down to 10-15, while overall accuracy on all methods did not dropped significantly. In fact, Naive Bayes performed even better that can be justified by reduced complexity of the probabilistic model. Also NF showed much better accuracy in comparison to other methods when using all features to distinguish between two malware datasets which can be linked to non-linear correlation in the data that are circumscribed in the Gaussian fuzzy patches.

Further, we investigated if there is any correlation between n-grams in files that belong to both benign and malicious classes. We extracted relative frequency of each n-gram according to the following formula $h_{n-\text { gram }}=\frac{N_{\text {files }}^{\text {Class }} n-\text { gram }}{N_{\text {files }}^{\text {Clas }}}$, where 
Table 6: Feature selection on 3-gram opcode features. Bold font denotes features that present in both datasets that include nenign samples

\begin{tabular}{|c|c|c|}
\hline Benign vs Malware_000 & Benign vs Malware 207 & Malware_000 vs Malware_207 \\
\hline \multicolumn{3}{|c|}{ Information Gain } \\
\hline merit attribute & merit attribute & merit attribute \\
\hline $\begin{array}{l}0.302483 \text { int3movpush } \\
0.283229 \text { int3int3mov } \\
0.266485 \text { popretint3 } \\
0.236949 \text { retint3int3 } \\
0.191866 \text { jmpint3int3 } \\
0.134709 \text { callmovtest } \\
0.133258 \text { movtestje } \\
0.115976 \text { callmovpop } \\
0.114482 \text { testjemov } \\
0.101328 \text { poppopret } \\
0.100371 \text { movtestjne }\end{array}$ & \begin{tabular}{|l|}
0.298812 int3movpush \\
0.279371 int3int3mov \\
0.227489 popretint3 \\
0.202162 retint3int3 \\
0.193938 jmpint3int3 \\
0.108580 retpushmov
\end{tabular} & $\begin{array}{l}0.042229 \text { pushlcallpushl } \\
0.039779 \text { movtestjne } \\
0.037087 \text { callpushlcall } \\
0.031045 \text { pushpushlcall }\end{array}$ \\
\hline \multicolumn{3}{|c|}{ Cfs } \\
\hline attribute & attribute & attribute \\
\hline $\begin{array}{l}\text { movtestje } \\
\text { callmovtest } \\
\text { callmovpop } \\
\text { retint3int3 } \\
\text { popretint3 } \\
\text { pushmovadd } \\
\text { int3int3mov } \\
\text { callmovjmp } \\
\text { jmpint3int3 } \\
\text { int3movpush }\end{array}$ & $\begin{array}{l}\text { movmovadd } \\
\text { retpushmov } \\
\text { xormovmov } \\
\text { callmovtest } \\
\text { popretint3 } \\
\text { pushmovadd } \\
\text { int3int3mov } \\
\text { callmovjmp } \\
\text { jmpint3int3 } \\
\text { int3movpush }\end{array}$ & $\begin{array}{l}\text { pushpushlcall } \\
\text { movtestjne } \\
\text { movmovjmp } \\
\text { jecmpje } \\
\text { cmpjepush } \\
\text { pushleacall } \\
\text { callpopret } \\
\text { leaveretpush } \\
\text { pushmovadd } \\
\text { pushcalllea } \\
\text { callpushlcall } \\
\text { callmovlea } \\
\text { pushlcallpushl } \\
\text { movmovmovl } \\
\text { calljmpmov }\end{array}$ \\
\hline
\end{tabular}

Table 7: Classification accuracy based on features from opcode 3-gram, in \%

\begin{tabular}{lccccccc}
\hline Dataset & Naive Bayes BayesNet & C4.5 & k-NN SVM & ANN & NF \\
\hline \multicolumn{7}{c}{ All features } \\
\hline Bn vs Ml_000 & 83.51 & 83.52 & $\mathbf{9 5 . 5 3}$ & 93.82 & 94.43 & 94.51 & 95.28 \\
Bn vs Ml_207 & 84.52 & 84.52 & $\mathbf{9 3 . 9 3}$ & 91.84 & 92.32 & 92.44 & 93.20 \\
Mn_000 vs Ml_207 & 63.73 & 63.73 & 81.21 & 78.64 & 75.42 & 76.64 & $\mathbf{8 3 . 1 3}$ \\
\hline \multicolumn{7}{c}{ Information Gain } \\
\hline Bn vs Ml_000 & 86.74 & 86.94 & 90.41 & $\mathbf{9 0 . 4 5}$ & 89.98 & 90.26 & 84.45 \\
Bn vs Ml_207 & 86.22 & 86.22 & 86.22 & 86.22 & 87.46 & $\mathbf{8 7 . 4 8}$ & 83.36 \\
Mn_000 vs Ml_207 & 63.19 & 62.55 & 71.19 & $\mathbf{7 1 . 8 9}$ & 69.54 & 67.36 & 69.14 \\
\hline \multicolumn{7}{c}{ Cfs } \\
\hline Bn vs Ml_000 & 87.79 & 88.66 & 91.15 & $\mathbf{9 1 . 2 2}$ & 90.90 & 90.82 & 85.31 \\
Bn vs Ml_207 & 86.24 & 86.33 & $\mathbf{8 9 . 9 2}$ & 89.73 & 89.17 & 89.34 & 81.58 \\
Mn_000 vs Ml_207 & 86.24 & 86.33 & $\mathbf{8 9 . 9 2}$ & 89.73 & 89.17 & 89.34 & 69.25 \\
\hline
\end{tabular}

$N_{\text {files } \in \text { - }}^{\text {Clasam }}$ indicates number of files in class that has n-gram and $N_{\text {files }}^{\text {Clas }}$ is a total number of files in this class. The results for 3-gram is depicted in the Figure 12. 
As a reference we took top 20 most frequent $\mathrm{n}$-grams from benign class and found frequency of the corresponding $\mathrm{n}$-grams from both malware datasets. It can be seen that the frequency does not differ fundamentally, yet n-grams for both malicious classes tend to have very close numbers in comparison to benign files. Moreover, there is a clear dependency between both malicious classes. We also can notice that most of the features selected from two datasets that includes benign samples are same. This highlights reliability of the selected 4-grams and generalization of this method for malware detection.

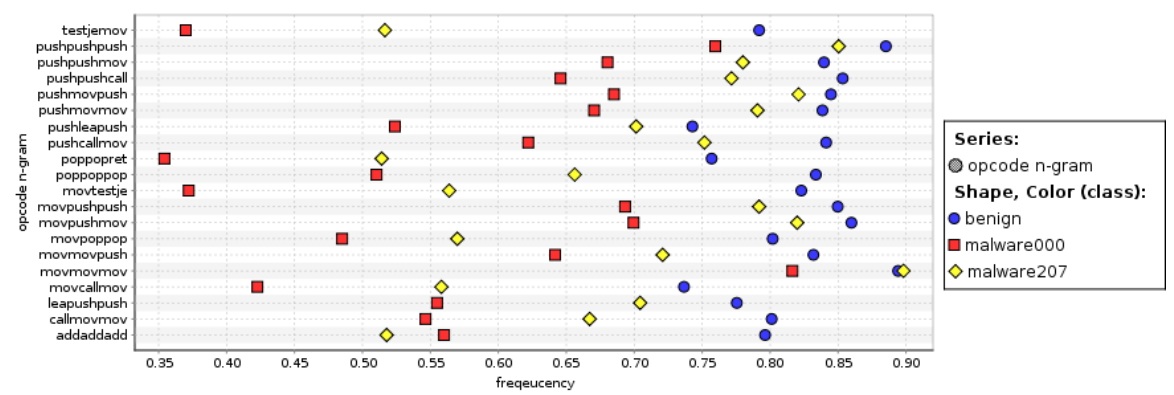

Fig. 12: Distribution of the frequencies of top 20 opcode 3-grams from benign set in comparison to both malicious datasets

Additionally, we studied 4-gram features and extracted 200 features as shown in Table 8. Similar to the 3 -grams features selected in the Table 6 one can see that two first pairs of datasets have a lot of common features, while the last one provides a significantly different set. As in case with 3-grams we used Information Gain with threshold of 0.1 for both benign and the first malware dataset, while for the last malware set we used InfoGain of 0.02, which looks reasonable with respect to number of selected' features.

The classification performance is given in Figure 9. As can be seen, 3-grams can show a bit better result than 4-grams in case of distinguishing between benign and malware_000 or Benign and malware_207 with C4.5 classifier. At the same time 4-grams are better in order to distinguish between two malware datasets with $\mathrm{C} 4.5$ classifier. We can conclude that results are quite good, and can be used for malware detection. In our opinion results can be improved by extracting more features and usage of relative frequencies rather than pure vectors.

In contrary to 3-grams we can see that the histograms of 4-grams have fundamental differences when it comes to malicious and benign sets as it is depicted in Figure 13. We can see that the frequencies correspond to malware_000 and malware_207 datasets are nearly similar and are far from the frequencies detected for the benign class. Moreover, there is a clear and strong correlation between two malware datasets. So, we can conclude that in case of probabilistic-based models like Bayes Network and Naive Bayes the classification could be a bit better due to differences in likelihood of appearance, which can be also found in Tables 7 and 9. 
Table 8: Feature selection on on 4-gram opcode features. Bold font denotes features that present in both datasets that include nenign samples

\begin{tabular}{|c|c|c|}
\hline Benign vs Malware_000 & Benign vs Malware_207 & Malware_000 vs Malware_207 \\
\hline \multicolumn{3}{|c|}{ Information Gain } \\
\hline merit attribute & merit attribute & merit attribute \\
\hline $\begin{array}{l}0.303209 \text { int3int3movpush } \\
0.295280 \text { int3movpushmov } \\
0.285608 \text { int3int3int3mov } \\
0.258733 \text { popretint3int3 } \\
0.241215 \text { poppopretint3 } \\
0.233205 \text { jmpint3int3int3 } \\
0.220679 \text { retint3int } 3 \text { int3 } \\
0.185178 \text { movpopretint3 } \\
0.151337 \text { movpushmovsub } \\
0.125703 \text { pushcallmovtest } \\
0.104993 \text { movpushmovpush } \\
0.104416 \text { movpushmovmov }\end{array}$ & \begin{tabular}{|l|}
0.295427 int3int3movpush \\
0.286378 int3movpushmov \\
0.266966 int3int3int3mov \\
0.229431 jmpint3int3int3 \\
0.224318 poppopretint3 \\
0.210289 popretint $3 i n t 3$ \\
0.170367 retint3int3int3 \\
0.148442 movpopretint3 \\
0.116760 movpushmovsub \\
0.103841 movpushmovpush \\
0.102730 movpushmovmov
\end{tabular} & $\begin{array}{l}0.047452 \text { pushlcallpushlcall } \\
0.045860 \text { movpoppopret } \\
0.044750 \text { jepushcallpop } \\
0.044573 \text { callpushlcallpushl } \\
0.038822 \text { cmpjepushcall } \\
0.035731 \text { pushcallpopret } \\
0.030460 \text { pushcallpopmov } \\
0.028564 \text { movcmpjepush } \\
0.025813 \text { cmpjecmpje } \\
0.024372 \text { leaveretpushmov } \\
0.023374 \text { pushpushpushlcall } \\
0.022312 \text { pushcallpoppop } \\
0.021929 \text { movtestjepush } \\
0.020003 \text { pushpushleapush }\end{array}$ \\
\hline \multicolumn{3}{|c|}{ Cfs } \\
\hline attribute & attribute & attribute \\
\hline $\begin{array}{l}\text { incaddincadd } \\
\text { movpushmovsub } \\
\text { jmpmovmovmov } \\
\text { pushcallmovtest } \\
\text { int3int3int3mov } \\
\text { movpoppopret } \\
\text { jmpint3int3int3 } \\
\text { movpopretint3 } \\
\text { int3int3movpush } \\
\text { int3movpushmov } \\
\text { poppopretint3 } \\
\text { addpushpushpush } \\
\text { pushpushcallea }\end{array}$ & $\begin{array}{l}\text { addaddaddadd } \\
\text { movmovpushpush } \\
\text { movpushmovsub } \\
\text { pushcallmovtest } \\
\text { int3int3int3mov } \\
\text { movxormovmov } \\
\text { pushlcallpushlcall } \\
\text { jmpint3int3int3 } \\
\text { movpopretint3 } \\
\text { int3int3movpush } \\
\text { int3movpushmov } \\
\text { poppopretint3 }\end{array}$ & $\begin{array}{l}\text { leaveretpushmov } \\
\text { callmovtestje } \\
\text { jepushcallpop } \\
\text { pushlcallpushlcall } \\
\text { pushpushpushlea } \\
\text { jecmpjecmp } \\
\text { movpoppopret } \\
\text { pushcallmovpush } \\
\text { pushmovmovcall } \\
\text { movpopretint3 } \\
\text { cmpjepushcall } \\
\text { movleamovmov } \\
\text { movmovjmpmov } \\
\text { pushpushcalllea } \\
\text { retnopnopnop } \\
\text { movaddpushpush } \\
\text { subpushpushpush }\end{array}$ \\
\hline
\end{tabular}

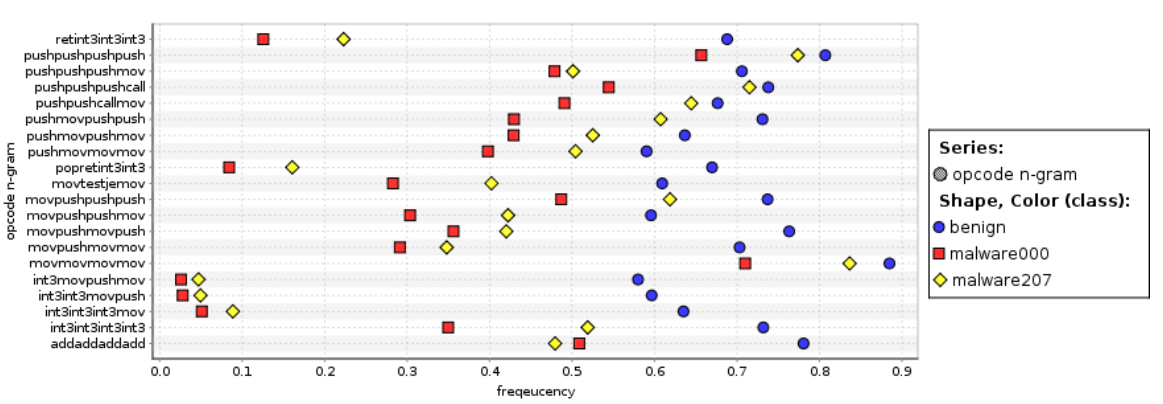

Fig. 13: Distribution of the frequencies of top 20 opcode 4-grams from benign set in comparison to both malicious datasets 
Table 9: Classification accuracy based on features from opcode 4-gram, in \%

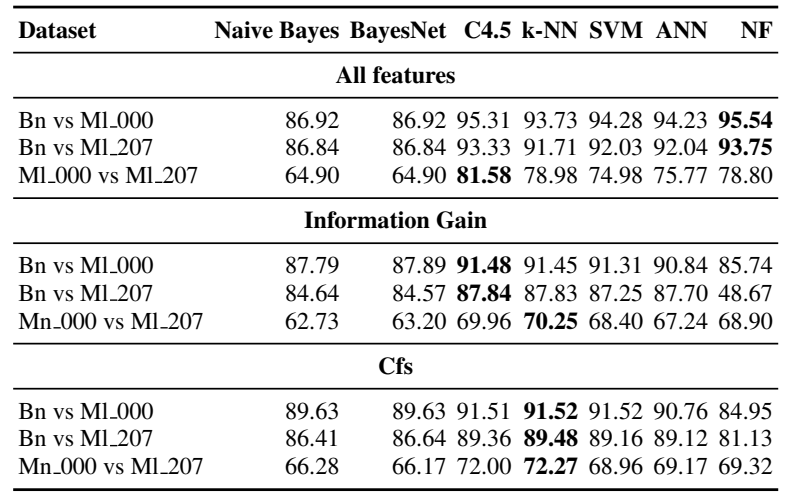

\subsubsection{API call n-grams}

API calls n-grams is the combination of specific operations invoked by the process in order to use functionalities of an operation system. In this study we used peframe to extract API calls from PE32 files. The bigger the n-gram size is the lower accuracy is possible to gain. The reason for this is that single API calls and their n-grams are far fewer in comparison with for example opcode n-grams. After extraction of API calls, we combined them into 1- and 2-grams. For each task we selected 100 most frequent features in a particular class and combined them into 200 -feature vectors. Tables 10 and 11 presents results of machine learning evaluation on API call $n$ grams data.

As we can see ANN, k-NN and C4.5 are the best classifiers similar to previous results. It is also more difficult to distinguish between files from malware_000 and malware_207. We gained quite high accuracy, but it is still lower than in related studies. It could be explained by the size of datasets: other studies datasets usually consist of several hundreds or thousands of files while our dataset has more than 110,000 files. After analysing feature selection results we decided not to include them in the results section since most of the features are similar in terms of distinguishing between malware and goodware. It means that there is large number of unique API calls that can be found once or twice in a file in contrary to the byte or opcode n-gram

Table 10: Classification accuracy based on API call 1-gram features, \%

\begin{tabular}{lccccccc}
\hline Dataset & Naive Bayes BayesNet & C4.5 & k-NN & SVM & ANN & NF \\
\hline \multicolumn{7}{c}{ All features } \\
\hline Bn vs Ml_000 & 90.79 & 90.79 & 93.39 & 93.47 & $\mathbf{9 3 . 5 1}$ & 93.43 & 82.44 \\
Bn vs Ml_207 & 87.18 & 87.18 & 90.94 & 91.03 & $\mathbf{9 1 . 3 7}$ & 91.23 & 81.28 \\
Ml_000 vs Ml_207 & 66.19 & 66.2 & $\mathbf{7 8 . 4 4}$ & 77.09 & 73.33 & 72.77 & 73.55 \\
\hline
\end{tabular}


Table 11: Classification accuracy based on API call 2-gram features, $\%$

\begin{tabular}{|c|c|c|c|c|}
\hline Dataset & Naive Bayes I & yesNet $\mathbf{C} 4.5$ & k-NN SVM ANN & NF \\
\hline \multicolumn{5}{|c|}{ All features } \\
\hline $\mathrm{Bn}$ & 86.54 & 86.5590 .88 & 91.5391 .9691 .85 & 75.24 \\
\hline Bn vs Ml_207 & 81.94 & 81.9187 .84 & $\begin{array}{llll}\mathbf{8 8 . 8 2} & 88.31 & 87.81\end{array}$ & 83.61 \\
\hline Ml_000 vs Ml_207 & 62.31 & 62.3173 .69 & $73.17 \quad 70.27 \quad 69.45$ & 70.08 \\
\hline
\end{tabular}

We also studied the difference between frequencies distributions of API calls. Figure 14 sketches extracted API 1-grams from three datasets. One can see that there is a significant spread between numbers of occurrences in benign class in contrary to both malicious datasets. On the other hand, results for both malware datasets are similar, which indicates statistical significance of extracted features. It is important ho highlight that the largest scatter are in frequencies for memset(), malloc() and free() API calls. On the other hand, malicious programs tend to use GetProcAddress() function more often for retrieving the address of any function from dynamic-link libraries in the system.

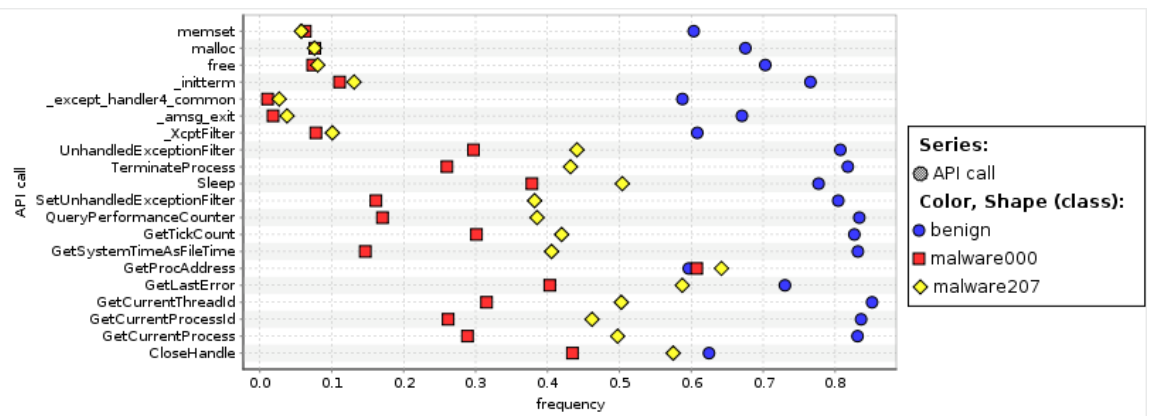

Fig. 14: frequencies of 20 most frequent API 1-grams for three different datasets

\section{Conclusion}

In this paper we presented a survey on applications of machine learning techniques for static analysis of PE32 Windows malware. First, we elaborated on different methods for extracting static characteristics of the executable files. Second, an overview of different machine learning methods utilized for classification of static characteristics of PE32 files was given. In addition, we offered a taxonomy of malware static features and corresponding ML methods. Finally, we provided a tutorial on how to apply different ML methods on benign and malware dataset for classification. We found that $\mathrm{C} 4.5$ and $\mathrm{k}-\mathrm{NN}$ in most cases perform better than other methods, 
while SVM and ANN on some feature sets showed good performance. On the other hand Bayes Network and Naive Bayes have poor performance compared to other ML methods. This can be explained by negligibly low probabilities which present in a large number of features such as opcode and bytes n-grams. So, it can see that static-analysis using ML is a fast and reliable mechanism to classify malicious and benign samples considering different characteristic of PE32 executables. Machine Learning-aided static malware analysis can be used as part of Cyber Threat Intelligence (CTI) activities to automate detection of indications of compromise from static features of PE32 Windows files.

\section{References}

1. Virusshare.com. http://virusshare.com/. accessed: 15.10.2015.

2. Vx heaven. http://vxheaven.org/. accessed: 25.10.2015.

3. Weka 3: Data mining software in java. http://www. cs. waikato.ac.nz/ml/weka/. accessed: 10.09.2015.

4. Gianni Amato. Peframe. https://github. com/guelfoweb/peframe. accessed: 20.10.2015.

5. M. Baig, P. Zavarsky, R. Ruhl, and D. Lindskog. The study of evasion of packed pe from static detection. In Internet Security (WorldCIS), 2012 World Congress on, pages 99-104, June 2012.

6. Simen Rune Bragen. Malware detection through opcode sequence analysis using machine learning. Master's thesis, Gjvik University College, 2015.

7. C. Cepeda, D. L. C. Tien, and P. Ordez. Feature selection and improving classification performance for malware detection. In 2016 IEEE International Conferences on Big Data and Cloud Computing (BDCloud), Social Computing and Networking (SocialCom), Sustainable Computing and Communications (SustainCom) (BDCloud-SocialCom-SustainCom), pages 560-566, Oct 2016.

8. Mohsen Damshenas, Ali Dehghantanha, and Ramlan Mahmoud. A survey on malware propagation, analysis, and detection. International Journal of Cyber-Security and Digital Forensics (IJCSDF), 2(4):10-29, 2013.

9. F. Daryabar, A. Dehghantanha, and N. I. Udzir. Investigation of bypassing malware defences and malware detections. In 2011 7th International Conference on Information Assurance and Security (IAS), pages 173-178, Dec 2011.

10. Farid Daryabar, Ali Dehghantanha, and Hoorang Ghasem Broujerdi. Investigation of malware defence and detection techniques. International Journal of Digital Information and Wireless Communications (IJDIWC), 1(3):645-650, 2011.

11. Farid Daryabar, Ali Dehghantanha, Brett Eterovic-Soric, and Kim-Kwang Raymond Choo. Forensic investigation of onedrive, box, googledrive and dropbox applications on android and ios devices. Australian Journal of Forensic Sciences, 48(6):615-642, 2016.

12. Farid Daryabar, Ali Dehghantanha, Nur Izura Udzir, Solahuddin bin Shamsuddin, et al. Towards secure model for scada systems. In Cyber Security, Cyber Warfare and Digital Forensic (CyberSec), 2012 International Conference on, pages 60-64. IEEE, 2012.

13. Farid Daryabar, Ali Dehghantanha, Nur Izura Udzir, et al. A review on impacts of cloud computing on digital forensics. International Journal of Cyber-Security and Digital Forensics (IJCSDF), 2(2):77-94, 2013.

14. Ali Dehghantanha and Katrin Franke. Privacy-respecting digital investigation. In Privacy, Security and Trust (PST), 2014 Twelfth Annual International Conference on, pages 129-138. IEEE, 2014. 
15. Dhruwajita Devi and Sukumar Nandi. Detection of packed malware. In Proceedings of the First International Conference on Security of Internet of Things, SecurIT '12, pages 22-26, New York, NY, USA, 2012. ACM.

16. Dennis Distler and Charles Hornat. Malware analysis: An introduction. Sans Reading Room, 2007.

17. T. Dube, R. Raines, G. Peterson, K. Bauer, M. Grimaila, and S. Rogers. Malware type recognition and cyber situational awareness. In Social Computing (SocialCom), 2010 IEEE Second International Conference on, pages 938-943, Aug 2010.

18. Tim Ebringer, Li Sun, and Serdar Boztas. A fast randomness test that preserves local detail. Virus Bulletin, 2008, 2008.

19. Parvez Faruki, Vijay Laxmi, M. S. Gaur, and P. Vinod. Mining control flow graph as api call-grams to detect portable executable malware. In Proceedings of the Fifth International Conference on Security of Information and Networks, SIN '12, pages 130-137, New York, NY, USA, 2012. ACM.

20. Anders Flaglien, Katrin Franke, and Andre Arnes. Identifying malware using cross-evidence correlation. In IFIP International Conference on Digital Forensics, pages 169-182. Springer Berlin Heidelberg, 2011.

21. Tristan Fletcher. Support vector machines explained. [Online]. http://sutikno. blog. undip. ac. id/files/2011/11/SVM-Explained. pdf.[Accessed 0606 2013], 2009.

22. Katrin Franke, Erik Hjelmås, and Stephen D Wolthusen. Advancing digital forensics. In IFIP World Conference on Information Security Education, pages 288-295. Springer Berlin Heidelberg, 2009.

23. Katrin Franke and Sargur N Srihari. Computational forensics: Towards hybrid-intelligent crime investigation. In Information Assurance and Security, 2007. IAS 2007. Third International Symposium on, pages 383-386. IEEE, 2007.

24. Mark A Hall and Lloyd A Smith. Practical feature subset selection for machine learning. Proceedings of the 21st Australasian Computer Science Conference ACSC'98, 1998.

25. Chris Hoffman. How to keep your pc secure when microsoft ends windows xp support. http://www.pcworld.com/article/2102606/ how-to-keep-your-pc-secure-when-microsoft-ends-windows-xp-support. html. accessed: 18.04.2016.

26. Anil K Jain, Robert PW Duin, and Jianchang Mao. Statistical pattern recognition: A review. Pattern Analysis and Machine Intelligence, IEEE Transactions on, 22(1):4-37, 2000.

27. Sachin Jain and Yogesh Kumar Meena. Byte level n-gram analysis for malware detection. In Computer Networks and Intelligent Computing, pages 51-59. Springer, 2011.

28. Kris Kendall and Chad McMillan. Practical malware analysis. In Black Hat Conference, USA, 2007.

29. Z. Khorsand and A. Hamzeh. A novel compression-based approach for malware detection using pe header. In Information and Knowledge Technology (IKT), 2013 5th Conference on, pages 127-133, May 2013.

30. Teuvo Kohonen and Timo Honkela. Kohonen network. Scholarpedia, 2(1):1568, 2007.

31. Jeremy Z. Kolter and Marcus A. Maloof. Learning to detect malicious executables in the wild. In Proceedings of the Tenth ACM SIGKDD International Conference on Knowledge Discovery and Data Mining, KDD '04, pages 470-478, New York, NY, USA, 2004. ACM.

32. Igor Kononenko and Matjaž Kukar. Machine learning and data mining: introduction to principles and algorithms. Horwood Publishing, 2007.

33. S. Kumar, M. Azad, O. Gomez, and R. Valdez. Can microsoft's service pack2 (sp2) security software prevent smurf attacks? In Advanced Int'l Conference on Telecommunications and Int'l Conference on Internet and Web Applications and Services (AICT-ICIW'06), pages 8989, Feb 2006.

34. Lastline. The threat of evasive malware. white paper, Lastline Labs, https:// www.lastline.com/papers/evasive_threats.pdf, February 2013. accessed: 29.10.2015. 
35. N. A. Le-Khac and A. Linke. Control flow change in assembly as a classifier in malware analysis. In 2016 4th International Symposium on Digital Forensic and Security (ISDFS), pages 38-43, April 2016.

36. Woody Leonhard. Atms will still run windows $\mathrm{xp}$ - but a bigger shift in security looms. http://www.infoworld.com/article/2610392/microsoft-windows/ atms-will-still-run-windows-xp----but-a-bigger-shift-in-security-looms . html, March 2014. accessed: 09.11.2015.

37. R. J. Mangialardo and J. C. Duarte. Integrating static and dynamic malware analysis using machine learning. IEEE Latin America Transactions, 13(9):3080-3087, Sept 2015.

38. Z. Markel and M. Bilzor. Building a machine learning classifier for malware detection. In Anti-malware Testing Research (WATeR), 2014 Second Workshop on, pages 1-4, Oct 2014.

39. M.M. Masud, L. Khan, and B. Thuraisingham. A hybrid model to detect malicious executables. In Communications, 2007. ICC '07. IEEE International Conference on, pages 14431448, June 2007.

40. Microsoft. Microsoft security essentials. http://windows.microsoft. com/en-us / windows/security-essentials-download. accessed: 18.04.2016.

41. Microsoft. Set application-specific access permissions. https://technet. microsoft.com/en-us/library/cc731858\%28v=ws.11\%29. aspx. accessed: 30.05.2016.

42. C. Miles, A. Lakhotia, C. LeDoux, A. Newsom, and V. Notani. Virusbattle: State-of-the-art malware analysis for better cyber threat intelligence. In 2014 7th International Symposium on Resilient Control Systems (ISRCS), pages 1-6, Aug 2014.

43. Nikola Milosevic, Ali Dehghantanha, and Kim-Kwang Raymond Choo. Machine learning aided android malware classification. Computers \& Electrical Engineering, 2017.

44. S. Naval, V. Laxmi, M. Rajarajan, M. S. Gaur, and M. Conti. Employing program semantics for malware detection. IEEE Transactions on Information Forensics and Security, 10(12):2591-2604, Dec 2015.

45. Farhood Norouzizadeh Dezfouli, Ali Dehghantanha, Brett Eterovic-Soric, and KimKwang Raymond Choo. Investigating social networking applications on smartphones detecting facebook, twitter, linkedin and google+ artefacts on android and ios platforms. Australian journal of forensic sciences, 48(4):469-488, 2016.

46. Opeyemi Osanaiye, Haibin Cai, Kim-Kwang Raymond Choo, Ali Dehghantanha, Zheng Xu, and Mqhele Dlodlo. Ensemble-based multi-filter feature selection method for ddos detection in cloud computing. EURASIP Journal on Wireless Communications and Networking, 2016(1):130, 2016.

47. Hamed Haddad Pajouh, Reza Javidan, Raouf Khayami, Dehghantanha Ali, and KimKwang Raymond Choo. A two-layer dimension reduction and two-tier classification model for anomaly-based intrusion detection in iot backbone networks. IEEE Transactions on Emerging Topics in Computing, 2016.

48. Shuhui Qi, Ming Xu, and Ning Zheng. A malware variant detection method based on byte randomness test. Journal of Computers, 8(10):2469-2477, 2013.

49. J. Ross Quinlan. Improved use of continuous attributes in c4. 5. Journal of artificial intelligence research, pages 77-90, 1996.

50. RC Quinlan. 4.5: Programs for machine learning morgan kaufmann publishers inc. San Francisco, USA, 1993.

51. D Krishna Sandeep Reddy and Arun K Pujari. N-gram analysis for computer virus detection. Journal in Computer Virology, 2(3):231-239, 2006.

52. Seth Rosenblatt. Malwarebytes: With anti-exploit, we'll stop the worst attacks on pcs. http://www.cnet.com/news/ malwarebytes-finally-unveils-freeware-exploit-killer/. accessed: 30.05 .2016 .

53. Neil J. Rubenking. The best antivirus utilities for 2016. http://uk.pcmag.com/antivirus-reviews/8141/guide/ the-best-antivirus-utilities-for-2016. accessed: 30.05.2016. 
54. Paul Rubens. 10 ways to keep windows $x p$ machines secure. http://www.cio.com/article/2376575/windows-xp/ 10-ways-to-keep-windows-xp-machines-secure.html. accessed: 18.04.2016.

55. Ashkan Sami, Babak Yadegari, Hossein Rahimi, Naser Peiravian, Sattar Hashemi, and Ali Hamze. Malware detection based on mining api calls. In Proceedings of the 2010 ACM Symposium on Applied Computing, SAC '10, pages 1020-1025, New York, NY, USA, 2010. ACM.

56. S. Samtani, K. Chinn, C. Larson, and H. Chen. Azsecure hacker assets portal: Cyber threat intelligence and malware analysis. In 2016 IEEE Conference on Intelligence and Security Informatics (ISI), pages 19-24, Sept 2016.

57. SANS. Who's using cyberthreat intelligence and how? https: //www.sans.org/reading-room/whitepapers/analyst/ cyberthreat-intelligence-how-35767. accessed: 01.03.2017.

58. Igor Santos, Felix Brezo, Xabier Ugarte-Pedrero, and Pablo G Bringas. Opcode sequences as representation of executables for data-mining-based unknown malware detection. Information Sciences, 231:64-82, 2013.

59. Igor Santos, Xabier Ugarte-Pedrero, Borja Sanz, Carlos Laorden, and Pablo G. Bringas. Collective classification for packed executable identification. In Proceedings of the 8th Annual Collaboration, Electronic Messaging, Anti-Abuse and Spam Conference, CEAS '11, pages 23-30, New York, NY, USA, 2011. ACM.

60. Asaf Shabtai, Yuval Fledel, and Yuval Elovici. Automated static code analysis for classifying android applications using machine learning. In Computational Intelligence and Security (CIS), 2010 International Conference on, pages 329-333. IEEE, 2010.

61. Kaveh Shaerpour, Ali Dehghantanha, and Ramlan Mahmod. Trends in android malware detection. The Journal of Digital Forensics, Security and Law: JDFSL, 8(3):21, 2013.

62. R.K. Shahzad, N. Lavesson, and H. Johnson. Accurate adware detection using opcode sequence extraction. In Availability, Reliability and Security (ARES), 2011 Sixth International Conference on, pages 189-195, Aug 2011.

63. Andrii Shalaginov and Katrin Franke. Automated generation of fuzzy rules from large-scale network traffic analysis in digital forensics investigations. In 7th International Conference on Soft Computing and Pattern Recognition (SoCPaR 2015). IEEE, 2015.

64. Andrii Shalaginov and Katrin Franke. A new method for an optimal som size determination in neuro-fuzzy for the digital forensics applications. In Advances in Computational Intelligence, pages 549-563. Springer International Publishing, 2015.

65. Andrii Shalaginov and Katrin Franke. A new method of fuzzy patches construction in neurofuzzy for malware detection. In IFSA-EUSFLAT. Atlantis Press, 2015.

66. Andrii Shalaginov and Katrin Franke. Automated intelligent multinomial classification of malware species using dynamic behavioural analysis. In IEEE Privacy, Security and Trust 2016, 2016.

67. Andrii Shalaginov and Katrin Franke. Big data analytics by automated generation of fuzzy rules for network forensics readiness. Applied Soft Computing, 2016.

68. Andrii Shalaginov and Katrin Franke. Towards Improvement of Multinomial Classification Accuracy of Neuro-Fuzzy for Digital Forensics Applications, pages 199-210. Springer International Publishing, Cham, 2016.

69. Andrii Shalaginov, Katrin Franke, and Xiongwei Huang. Malware beaconing detection by mining large-scale dns logs for targeted attack identification. In 18th International Conference on Computational Intelligence in Security Information Systems. WASET, 2016.

70. Andrii Shalaginov, Lars Strande Grini, and Katrin Franke. Understanding neuro-fuzzy on a class of multinomial malware detection problems. In IEEE International Joint Conference on Neural Networks (IJCNN 2016), Jul 2016.

71. M. Shankarapani, K. Kancherla, S. Ramammoorthy, R. Movva, and S. Mukkamala. Kernel machines for malware classification and similarity analysis. In Neural Networks (IJCNN), The 2010 International Joint Conference on, pages 1-6, July 2010. 
72. Muazzam Ahmed Siddiqui. Data mining methods for malware detection. ProQuest, 2008.

73. Holly Stewart. Infection rates and end of support for windows $x$. https://blogs.technet.microsoft.com/mmpc/2013/10/29/ infection-rates-and-end-of-support-for-windows-xp/. accessed: 01.04.2016.

74. Li Sun, Steven Versteeg, Serdar Boztaş, and Trevor Yann. Pattern recognition techniques for the classification of malware packers. In Information security and privacy, pages 370-390. Springer, 2010.

75. S Momina Tabish, M Zubair Shafiq, and Muddassar Farooq. Malware detection using statistical analysis of byte-level file content. In Proceedings of the ACM SIGKDD Workshop on CyberSecurity and Intelligence Informatics, pages 23-31. ACM, 2009.

76. Shugang Tang. The detection of trojan horse based on the data mining. In Fuzzy Systems and Knowledge Discovery, 2009. FSKD '09. Sixth International Conference on, volume 1, pages 311-314, Aug 2009.

77. X. Ugarte-Pedrero, I. Santos, P.G. Bringas, M. Gastesi, and J.M. Esparza. Semi-supervised learning for packed executable detection. In Network and System Security (NSS), 2011 5th International Conference on, pages 342-346, Sept 2011.

78. R Veeramani and Nitin Rai. Windows api based malware detection and framework analysis. In International conference on networks and cyber security, volume 25, 2012.

79. C. Wang, Z. Qin, J. Zhang, and H. Yin. A malware variants detection methodology with an opcode based feature method and a fast density based clustering algorithm. In 2016 12th International Conference on Natural Computation, Fuzzy Systems and Knowledge Discovery (ICNC-FSKD), pages 481-487, Aug 2016.

80. Tzu-Yen Wang, Chin-Hsiung Wu, and Chu-Cheng Hsieh. Detecting unknown malicious executables using portable executable headers. In INC, IMS and IDC, 2009. NCM '09. Fifth International Joint Conference on, pages 278-284, Aug 2009.

81. Steve Watson and Ali Dehghantanha. Digital forensics: the missing piece of the internet of things promise. Computer Fraud \& Security, 2016(6):5-8, 2016.

82. Yanfang Ye, Dingding Wang, Tao Li, Dongyi Ye, and Qingshan Jiang. An intelligent pemalware detection system based on association mining. Journal in computer virology, 4(4):323-334, 2008.

83. M.N.A. Zabidi, M.A. Maarof, and A. Zainal. Malware analysis with multiple features. In Computer Modelling and Simulation (UKSim), 2012 UKSim 14th International Conference on, pages 231-235, March 2012.

84. Zongqu Zhao. A virus detection scheme based on features of control flow graph. In Artificial Intelligence, Management Science and Electronic Commerce (AIMSEC), 2011 2nd International Conference on, pages 943-947, Aug 2011. 